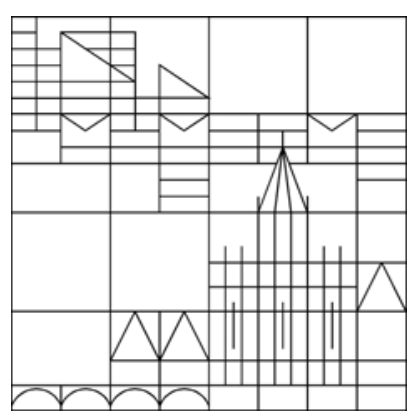

\title{
Importance sampling for backward SDEs
}

\author{
Christian Bender \\ Thilo Moseler
}

Konstanzer Schriften in Mathematik und Informatik

Nr. 254, Oktober 2008

ISSN 1430-3558

Konstanzer Online-Publikations-System (KOPS)

URL: http://www.ub.uni-konstanz.de/kops/volltexte/2008/6522/

URN: http://nbn-resolving.de/urn:nbn:de:bsz:352-opus-65227

\author{
(C) Fachbereich Mathematik und Statistik \\ (C) Fachbereich Informatik und Informationswissenschaft \\ Universität Konstanz \\ Fach D 188, 78457 Konstanz, Germany \\ E-Mail: preprints@informatik.uni-konstanz.de \\ WWW: http://www.informatik.uni-konstanz.de/Schriften/
}





\title{
Importance sampling for backward SDEs
}

\author{
Christian Bender ${ }^{a}$, Thilo Moseler ${ }^{b}$
}

September 19, 2008

\author{
${ }^{a}$ Institute for Mathematical Stochastics, TU Braunschweig, Pockelsstr. 14, D-38107 Braunschweig, \\ Germany, C.Bender@tu-bs.de \\ ${ }^{b}$ Corresponding author: Department for Mathematics and Statistics, University of Konstanz, D-78457 \\ Konstanz, Germany, Thilo.Moseler@uni-konstanz.de
}

\begin{abstract}
In this paper we explain how the importance sampling technique can be generalized from simulating expectations to computing the initial value of backward SDEs with Lipschitz continuous driver. By means of a measure transformation we introduce a variance reduced version of the forward approximation scheme by Bender and Denk [4] for simulating backward SDEs. A fully implementable algorithm using the least-squares Monte Carlo approach is developed and its convergence is proved. The success of the generalized importance sampling is illustrated by numerical examples in the context of Asian option pricing under different interest rates for borrowing and lending.
\end{abstract}

Keywords: BSDE, Numerics, Monte Carlo simulation, Variance reduction

AMS classification: 65C30, 65C05, 91B28

\section{Introduction}

The solutions of a variety of optimal portfolio selection problems and option pricing problems from mathematical finance can be represented via backward stochastic differential equations (BSDEs), driven by a Brownian motion $W$, of the form

$$
\begin{aligned}
d S_{t} & =b\left(t, S_{t}\right) d t+\sigma\left(t, S_{t}\right) d W_{t}, & & S_{0}=s_{0}, \\
d Y_{t} & =-f\left(t, S_{t}, Y_{t}, Z_{t}\right) d t+Z_{t} d W_{t}, & & Y_{T}=\Phi(S) .
\end{aligned}
$$

In the context of option pricing, $S$ typically is a basket of financial underlyings, $\Phi$ is the payoff function of the option, $Y$ is the price process of the option, and $Z$ is related to a hedging strategy (possibly in the Föllmer-Schweizer sense), see e.g. the survey article by El Karoui et al. [11]. In the classical pricing problem of options without early-exercise features, the driver $f$ is linear and so today's price $Y_{0}$ reduces to the expectation of the discounted option payoff under an equivalent martingale measure. In general, the driver may become nonlinear, for example when considering different interest rates for borrowing and investing in a bond, see Bergman [6], or when computing utility indifference prices, see e.g. Becherer [2].

In the classical linear option pricing problem, a generic way to calculate prices numerically is to apply a Monte Carlo simulation of the underlyings and then average over the discounted payoffs. However, the 
estimators for the option prices resulting out of this procedure often suffer from high empirical variance. This is, in particular, the case for out-of-the-money options or more general for options containing some rare-event feature.

The efficiency of the Monte Carlo approach may be drastically increased by the choice of an appropriate variance reduction technique. In this respect the importance sampling technique turns out to be highly efficient for some path dependent options, for instance of Asian type, see e.g. Glasserman [13]. The basic idea of importance sampling is to change the drift of the underlyings by a change of measure in order to force more simulated paths to take value in 'interesting' regions (e.g. in the money). In this way one obtains more non-zero pay-offs resulting in a more stable estimator. One delicate feature of importance sampling is its requirement for tailor-made choices for the new measure. Choosing a wrong drift rather results in variance blow-up than in variance reduction. The complexity of this method is reflected by the vast existing literature concerning the 'optimal choice' of the new measure in diffusion models. While one branch of literature tries to tackle the problem in continuous time, see e.g. the articles of Newton [23], Milstein and Schoenmakers [22] or Guasoni and Robertson [16], other authors develop specific strategies for special settings in discrete time, see e.g. Boyle et al. [8], Glasserman et al. [12] or Ökten et al. [24]. Besides its application in finance importance sampling methods are also used in many other areas such as environmental modelling [25], biology [15], or computer graphics [26].

The aim of the present paper is to introduce importance sampling to Monte Carlo schemes for nonlinear pricing problems which are represented by nonlinear BSDEs. There is by now a variety of Monte Carlo schemes for BSDEs which can be distinguished by two features: Firstly a scheme can be directed backwardly in time as the ones suggested by Gobet et al. [14, 19], Bouchard and Touzi [7], and Zhang [27], or forwardly through Picard iterations as proposed by Bender and Denk [4] and Labart [18], Ch. III. Secondly the schemes differ by the kind of Monte Carlo estimator which is applied to approximate the nested conditional expectations. Popular choices are estimators based on Malliavin calculus [7], nonparametric regression [18], quantization [1, 10], and least-squares Monte Carlo [4, 5, 14, 19]. We briefly mention that least-squares Monte Carlo has also been successfully applied to the pricing problem of early exercise options, see $[3,9,20]$.

In this paper we focus on the forward scheme with least-squares Monte Carlo, i.e. we introduce importance sampling in the context of the paper by Bender and Denk [4], but it is straightforward how the ideas can, in principle, be transferred to the other settings. The paper is organized as follows: After setting the problem we briefly resume the Picard-type scheme of Bender and Denk in Section 2. Section 3 introduces a modified version of this forward working technique. Parameterized by a change of measure we introduce several time discretizations for $\left(Y_{0}, Z_{0}\right)$ and analyze the error due to the time discretization and the Picard iteration. We then replace the conditional expectations by a least-squares Monte Carlo estimator in Section 4. Here the change of measure for the importance sampling considerably complicates the situation, as the approximations for $(Y, Z)$ need not be square integrable under the original measure. To get around this difficulty, it is essential to carefully take the density process of the change of measure into account, when designing an appropriate regression basis. We analyze the regression error in dependence on the choice of basis and prove convergence of the corresponding Monte Carlo estimator as the number of simulated paths tends to infinity. Finally we demonstrate the success of the variance reduced estimator in a simulation study in the context of Asian option pricing under different interest rates in a Black-Scholes economy. In this study we find a variance reduction of more than factor 10 for the at-the-money case and more than factor 35 for the out-of-the-money case.

\section{Preliminaries}

We investigate numerical solutions of the following decoupled forward-backward stochastic differential equation $(\mathrm{FBSDE})$ on a complete probability space $\left(\Omega, \mathcal{F}, \mathcal{F}_{t}, P\right)$, where the filtration $\left(\mathcal{F}_{t}\right)$ is the aug- 
mentation of the one generated by a $D$-dimensional Brownian motion $W$ :

$$
\begin{aligned}
d S_{t} & =b\left(t, S_{t}\right) d t+\sigma\left(t, S_{t}\right) d W_{t}, & & S_{0}=s_{0}, \\
d Y_{t} & =-f\left(t, S_{t}, Y_{t}, Z_{t}\right) d t+Z_{t} d W_{t}, & & Y_{T}=\Phi(S) .
\end{aligned}
$$

Here the coefficient functions $b:[0, T] \times \mathbb{R}^{M} \longrightarrow \mathbb{R}^{M}, \sigma:[0, T] \times \mathbb{R}^{M} \longrightarrow \mathbb{R}^{M \times D}, f:[0, T] \times \mathbb{R}^{M} \times \mathbb{R} \times$ $\mathbb{R}^{D} \longrightarrow \mathbb{R}$ are given. The terminal condition for the BSDE is defined via the functional $\Phi$, which acts on the paths of $S$ and is Lipschitz continuous in the sup-norm. Recall, that a solution is a triplet $(S, Y, Z)$ of $\left(\mathcal{F}_{t}\right)$-adapted, square-integrable stochastic processes. We require throughout this paper the following assumptions, which in particular ensure the existence of a unique solution:

A 1. For each $(t, s),\left(t^{\prime}, s^{\prime}\right) \in\left([0, T] \times \mathbb{R}^{M}\right)$ :

$$
\left|b(t, s)-b\left(t^{\prime}, s^{\prime}\right)\right|+\left|\sigma(t, s)-\sigma\left(t^{\prime}, s^{\prime}\right)\right| \leq K\left(\sqrt{\left|t-t^{\prime}\right|}+\left|s-s^{\prime}\right|\right) .
$$

A 2. For each $(t, s, y, z),\left(t^{\prime}, s^{\prime}, y^{\prime}, z^{\prime}\right) \in\left([0, T] \times \mathbb{R}^{M} \times \mathbb{R} \times \mathbb{R}^{D}\right)$ :

$$
\left|f(t, s, y, z)-f\left(t^{\prime}, s^{\prime}, y^{\prime}, z^{\prime}\right)\right| \leq K\left(\sqrt{\left|t-t^{\prime}\right|}+\left|s-s^{\prime}\right|+\left|y-y^{\prime}\right|+\left|z-z^{\prime}\right|\right) .
$$

A 3. There is an $M^{\prime}$-dimensional Markov process $\left(X_{t}, \mathcal{F}_{t}\right)$ with $S_{t}$ as its first $M$ components such that

$$
E\left[\sup _{0 \leq t \leq T}\left|X_{t}\right|^{2}\right]<\infty
$$

and $\Phi(S)=\phi\left(X_{T}\right)$ for some Lipschitz continuous function $\phi$ with Lipschitz constant $K$.

A 4.

$$
\sup _{0 \leq t \leq T}|b(t, 0)|+|\sigma(t, 0)|+|f(t, 0,0,0)|+|\phi(0)| \leq K .
$$

We now explain the starting point for the algorithm developed later on. Consider the following family of decoupled FBSDEs parameterized by some measurable, bounded and adapted process $h:[0, T] \longrightarrow \mathbb{R}^{D}$ :

$$
\begin{aligned}
d S_{t}^{h} & =\left[b\left(t, S_{t}^{h}\right)+\sigma\left(t, S_{t}^{h}\right) h_{t}\right] d t+\sigma\left(t, S_{t}^{h}\right) d W_{t} \\
d Y_{t}^{h} & =\left[-f\left(t, S_{t}^{h}, Y_{t}^{h}, Z_{t}^{h}\right)+\left(Z_{t}^{h}\right)^{\top} h_{t}\right] d t+Z_{t}^{h} d W_{t} \\
S_{0}^{h} & =s_{0}, \quad Y_{T}^{h}=\phi\left(X_{T}^{h}\right) .
\end{aligned}
$$

where ${ }^{\top}$ denotes the transposition of a matrix. We denote $(S, Y, Z):=\left(S^{0}, Y^{0}, Z^{0}\right)$, the solution of the original FBSDE with $h \equiv 0$.

The first observation is that the initial value of the backward part does not depend on $h$. In fact, defining a new measure $Q^{h}$ by $d Q^{h}=\Psi_{T}^{h} d P$ where

$$
\Psi_{t}^{h}=\exp \left\{-\int_{0}^{t} h_{u}^{\top} d W_{u}-\frac{1}{2} \int_{0}^{t}\left|h_{u}\right|^{2} d u\right\},
$$

we can apply the Girsanov theorem, to deduce that the law of $\left(S^{h}, Y^{h}, Z^{h}\right)$ under $Q^{h}$ is the same as that of $(S, Y, Z)$ under $P$. In particular, the constants $\left(Y_{0}, Z_{0}\right)$ and $\left(Y_{0}^{h}, Z_{0}^{h}\right)$ coincide. We mention that, however, the path of the processes at later time points $\left(S^{h}, Y^{h}, Z^{h}\right)$ and $(S, Y, Z)$ differ. Nonetheless, in many applications, e.g. in option pricing problems, one is mainly interesting in estimating $Y_{0}$. Having the different representations for $Y_{0}$ at hand, we aim at reducing the variance of Monte Carlo estimators for $Y_{0}$ by a judicious choice of $h$. This turns out to generalize the importance sampling technique from calculating expectations to nonlinear BSDEs. In the present paper we concentrate on a specific Monte 
Carlo scheme for BSDEs, namely the forward scheme by Bender and Denk [4], which we now briefly review. Generalization to other Monte Carlo schemes for BSDEs are expected to be straightforward.

For a given partition $\pi: 0=t_{0}<\ldots<t_{N}=T$ with $\sup _{i}\left|t_{i+1}-t_{i}\right|=:|\pi|<1$ we define $\Delta_{i}=t_{i+1}-t_{i}$ and use the Euler-Maruyama scheme $S^{\pi}$ for the forward part of the system $S$. The increments of the Brownian motion are denoted by $\Delta W_{i}=W_{i+1}-W_{i}$. We add the following assumption:

A 5. For every partition $\pi$ there is a deterministic function $u_{\pi}: \pi \times \mathbb{R}^{M^{\prime}} \times \mathbb{R}^{D} \longrightarrow \mathbb{R}^{M^{\prime}}$ such that

$$
X_{t_{i}}^{\pi}=u_{\pi}\left(t_{i}, X_{t_{i-1}}^{\pi}, \Delta W_{i-1}^{\pi}\right), \quad X_{t_{0}}^{\pi}=X_{0},
$$

satisfies $X_{m, t_{i}}^{\pi}=S_{m, t_{i}}^{\pi}$ for $m \leq M$ and $E\left[\left|X_{t_{N}}^{\pi}-X_{T}\right|^{2}\right] \longrightarrow 0$ as $|\pi| \longrightarrow 0$.

Under Assumption A $5\left(X_{t_{i}}^{\pi}, \mathcal{F}_{t_{i}}\right)$ is a Markov process under $P$ as well.

The approximation scheme for the backward part is now defined recursively for $0 \leq i \leq N$ by

$$
\begin{aligned}
Y_{t_{i}}^{n, \pi} & =E\left[\phi\left(X_{T}^{\pi}\right)+\sum_{j=i}^{N-1} f\left(t_{j}, S_{t_{j}}^{\pi}, Y_{t_{j}}^{n-1, \pi}, Z_{t_{j}}^{n-1, \pi}\right) \Delta_{j} \mid \mathcal{F}_{t_{i}}\right] \\
Z_{d, t_{i}}^{n, \pi} & =E\left[\frac{\Delta W_{d, i}}{\Delta_{i}}\left(\phi\left(X_{T}^{\pi}\right)+\sum_{j=i+1}^{N-1} f\left(t_{j}, S_{t_{j}}^{\pi}, Y_{t_{j}}^{n-1, \pi}, Z_{t_{j}}^{n-1, \pi}\right) \Delta_{j}\right) \mid \mathcal{F}_{t_{i}}\right] .
\end{aligned}
$$

initialized at $\left(Y^{0, \pi}, Z^{0, \pi}\right)=(0,0)$. We apply the convention $\Delta W_{N}:=0$ and use constant extensions for the approximation, i.e. $Y_{t}^{n, \pi}:=Y_{t_{i}}^{n, \pi}$ and $Z_{t}^{n, \pi}:=Z_{t_{i}}^{n, \pi}$ for $t \in\left[t_{i}, t_{i+1}[\right.$.

Theorem 2 of Bender and Denk [4] gives the convergence of the Picard-type discretization scheme:

Theorem 2.1. There is a constant $C$ such that

$$
\sup _{0 \leq t \leq T} E\left[\left|Y_{t}-Y_{t}^{n, \pi}\right|^{2}\right]+E\left[\int_{0}^{T}\left|Z_{s}-Z_{s}^{n, \pi}\right|^{2} d s\right] \leq C E\left[\left|X_{T}-X_{t_{N}}^{\pi}\right|^{2}\right]+C|\pi|+C\left(\frac{1}{2}+C^{*}|\pi|\right)^{n}
$$

where $C^{*}=K^{2}(T+1)\left(4 D K^{2}(T+1) D T+1\right)$.

In comparison to the backward schemes of Bouchard and Touzi [7], Gobet et al. [14] and Zhang [27] the error estimate contains an extra term due to the Picard iterations. This drawback is offset by the moderate error occurring by the approximation of the conditional expectation with some estimator. The error in the forward scheme does not explode if the mesh grid size tends to zero as it is the case for the backward schemes. For more details, see the discussion in [4], pp. 1802-1803.

\section{Modified forward scheme}

In this section we introduce the time discretized analogue to the Picard-type iteration scheme with importance sampling induced by some process $h$. As it is natural that the choice of $h$ will vary with the partition $\pi$, we do assume from now on that the partition $\pi$ is fixed. At first we specify the class of processes which we will consider in the sequel.

A 6. The discretized process $h$ is given by

$$
h_{t_{i}}=\widetilde{h}\left(t_{i}, \Delta W_{0}, \ldots, \Delta W_{i-1}\right)
$$

for some bounded deterministic function $\widetilde{h}: \pi \times \mathbb{R}^{D} \times \ldots \times \mathbb{R}^{D} \longrightarrow \mathbb{R}^{D}$. The bound of $h$ will be denoted $C_{h}$. 
The modified forward scheme is then given by

$$
\begin{aligned}
\Delta W_{i}^{h, \pi} & =\Delta W_{i}+h_{t_{i}} \Delta_{i}, 0 \leq i \leq N-1, \quad \Delta W_{N}^{h, \pi}=0, \\
\Psi_{t_{i}}^{h, \pi, j} & =\exp \left\{-\sum_{k=j}^{i-1} h_{t_{k}}^{\top} \Delta W_{k}-\frac{1}{2} \sum_{k=j}^{i-1}\left|h_{t_{k}}\right|^{2} \Delta_{k}\right\}, \quad 0 \leq j \leq i \leq N, \\
X_{t_{0}}^{h, \pi} & =X_{0} \\
X_{t_{i}}^{h, \pi} & =u_{\pi}\left(t_{i}, X_{t_{i-1}}^{h, \pi}, \Delta W_{i-1}^{h, \pi}\right), \quad 1 \leq i \leq N,
\end{aligned}
$$

and, for $0 \leq i \leq N$,

$$
\begin{aligned}
Y_{t_{i}}^{h, n, \pi} & \left.=E\left[\Psi_{t_{N}}^{h, \pi, i} \phi\left(X_{t_{N}}^{h, \pi}\right)+\sum_{j=i}^{N-1} \Psi_{t_{j}}^{h, \pi, i} f\left(t_{j}, S_{t_{j}}^{h, \pi}, Y_{t_{j}}^{h, n-1, \pi}, Z_{t_{j}}^{h, n-1, \pi}\right) \Delta_{j}\right) \mid \mathcal{F}_{t_{i}}\right] \\
Z_{d, t_{i}}^{h, n} & =E\left[\frac{\Delta W_{d, i}^{h, \pi}}{\Delta_{i}}\left(\Psi_{t_{N}}^{h, \pi, i} \Phi\left(S^{h, \pi}\right)+\sum_{j=i+1}^{N-1} \Psi_{t_{j}}^{h, \pi, i} f\left(t_{j}, S_{t_{j}}^{h, \pi}, Y_{t_{j}}^{h, n-1, \pi}, Z_{t_{j}}^{h, n-1, \pi}\right) \Delta_{j}\right) \mid \mathcal{F}_{t_{i}}\right],
\end{aligned}
$$

initialized at $\left(Y_{t_{i}}^{h, 0, \pi}, Z_{t_{i}}^{h, 0, \pi}\right)=(0,0)$. Again, we omit the superscript $h$, if $h \equiv 0$, in which case this is just the forward scheme discussed in Section 2. Note that, by construction, the first $M$ components of $X_{t_{i}}^{h, \pi}$ coincide with $S_{t_{i}}^{h, \pi}$ defined via the Euler-Maruyama scheme

$$
\begin{aligned}
S_{t_{0}}^{h, \pi} & =s_{0}, \\
S_{t_{i+1}}^{h, \pi} & =\left[b\left(t_{i}, S_{t_{i}}^{h, \pi}\right)+\sigma\left(t_{i}, S_{t_{i}}^{h, \pi}\right) h_{t_{i}}\right] \Delta_{i}+\sigma\left(t_{i}, S_{t_{i}}^{h, \pi}\right) \Delta W_{i}, \quad 0 \leq i \leq N-1 .
\end{aligned}
$$

Defining a new measure $Q^{h, \pi}$ by $d Q^{h, \pi}=\Psi_{t_{N}}^{h, \pi, 0} d P$ the Girsanov theorem implies that the process

$$
W_{t}^{h, \pi}=W_{t}+\sum_{j=0}^{N-1} h_{t_{j}}\left(t_{j+1} \wedge t-t_{j} \wedge t\right),
$$

is Brownian motion under $Q^{h, \pi}$. Consequently, $\Delta W^{h, \pi}$ are Brownian increments under this measure. This implies that $\left(X^{h, \pi}, \mathcal{F}_{t_{i}}\right)$ is a Markovian process under $Q^{h, \pi}$ and that the transition probabilities of $X^{h, \pi}$ under $Q^{h, \pi}$ are the same as those of $X^{\pi}$ under $P$.

The following theorem shows that, in this Markovian setting, the conditional expectations in the above iteration scheme actually simplify to regressions on $X_{t_{i}}^{h, \pi}$. On the one hand this is crucial for the Monte Carlo algorithm described in the next section, on the other hand it also allows us to derive some convergence results for the modified scheme in an elegant way.

Theorem 3.1. Under the standing assumptions there are deterministic functions $y_{i}^{n, \pi}$ and $z_{i}^{n, \pi}$ not depending on $h$ such that

$$
\begin{gathered}
Y_{t_{i}}^{h, n, \pi}=y_{i}^{n, \pi}\left(X_{t_{i}}^{h, \pi}\right), \\
Z_{t_{i}}^{h, n, \pi}=z_{i}^{n, \pi}\left(X_{t_{i}}^{h, \pi}\right) .
\end{gathered}
$$

In particular,

$$
\begin{aligned}
Y_{t_{i}}^{h, n, \pi} & =E\left[\Psi_{t_{N}}^{h, \pi, i} \phi\left(X_{t_{N}}^{h, \pi}\right)+\sum_{j=i}^{N-1} \Psi_{t_{j}}^{h, \pi, i} f\left(t_{j}, S_{t_{j}}^{h, \pi}, Y_{t_{j}}^{h, n-1, \pi}, Z_{t_{j}}^{h, n-1, \pi}\right) \Delta_{j} \mid X_{t_{i}}^{h, \pi}\right], \\
Z_{d, t_{i}}^{h, n, \pi} & =E\left[\frac{\Delta W_{d, i}^{h, \pi}}{\Delta_{i}}\left(\Psi_{t_{N}}^{h, \pi, i} \phi\left(X_{t_{N}}^{h, \pi}\right)+\sum_{j=i+1}^{N-1} \Psi_{t_{j}}^{h, \pi, i} f\left(t_{j}, S_{t_{j}}^{h, \pi}, Y_{t_{j}}^{h, n-1, \pi}, Z_{t_{j}}^{h, n-1, \pi}\right) \Delta_{j}\right) \mid X_{t_{i}}^{h, \pi}\right] .
\end{aligned}
$$


Proof. We proceed with a double induction, working forward in Picard-iterations and backward in time. The claim is true for $n=0, i=0, \ldots, N$, since by definition $Y_{t_{i}}^{h, 0, \pi}=0=Z_{d, t_{i}}^{h, 0, \pi}$ for $d=1, \ldots, D$. Due to the terminal condition $Y_{t_{N}}^{h, n, \pi}=\phi\left(X_{t_{N}}^{h, \pi}\right)$ and $Z_{t_{N}}^{h, n, \pi}=0$ for each $n$ it is also valid for $n \in \mathbb{N}$ and $i=N$.

Now, suppose the claim is true for $Y^{h, n-1, \pi}, Z^{h, n-1, \pi}$ and for $Y_{t_{i+1}}^{h, n, \pi}, Z_{t_{i+1}}^{h, n, \pi}$, for some $i \leq N-1$. Then we can conclude

$$
\begin{aligned}
Y_{t_{i}}^{h, n, \pi} & =E\left[\Psi_{t_{N}}^{h, \pi, i} \phi\left(X_{t_{N}}^{h, \pi}\right)+\sum_{j=i}^{N-1} \Psi_{t_{j}}^{h, \pi, i} f\left(t_{j}, S_{t_{j}}^{h, \pi}, Y_{t_{j}}^{h, n-1, \pi}, Z_{t_{j}}^{h, n-1, \pi}\right) \Delta_{j} \mid \mathcal{F}_{t_{i}}\right] \\
& =E\left[\Psi_{t_{N}}^{h, \pi, i} \phi\left(X_{t_{N}}^{h, \pi}\right)+\sum_{j=i}^{N-1} E\left[\Psi_{t_{N}}^{h, \pi, i} \mid \mathcal{F}_{t_{j}}\right] f\left(t_{j}, S_{t_{j}}^{h, \pi}, Y_{t_{j}}^{h, n-1, \pi}, Z_{t_{j}}^{h, n-1, \pi}\right) \Delta_{j} \mid \mathcal{F}_{t_{i}}\right] \\
& =E\left[\Psi_{t_{N}}^{h, \pi, i}\left(\phi\left(X_{t_{N}}^{h, \pi}\right)+\sum_{j=i}^{N-1} f\left(t_{j}, S_{t_{j}}^{h, \pi}, Y_{t_{j}}^{h, n-1, \pi}, Z_{t_{j}}^{h, n-1, \pi}\right) \Delta_{j}\right) \mid \mathcal{F}_{t_{i}}\right] \\
& =E^{Q^{h, \pi}}\left[Y_{t_{i+1}}^{h, n, \pi} \mid \mathcal{F}_{t_{i}}\right]+f\left(t_{i}, S_{t_{i}}^{h, \pi}, Y_{t_{i}}^{h, n-1, \pi}, Z_{t_{i}}^{h, n-1, \pi}\right) \Delta_{i} \\
& =E^{Q^{h, \pi}}\left[y_{i+1}^{n, \pi}\left(X_{t_{i+1}}^{h, \pi}\right) \mid \mathcal{F}_{t_{i}}\right]+f\left(t_{i}, S_{t_{i}}^{h, \pi}, y_{i}^{n-1, \pi}\left(X_{t_{i}}^{h, \pi}\right), z_{i}^{n-1, \pi}\left(X_{t_{i}}^{h, \pi}\right)\right) \Delta_{i} \\
& =E^{Q^{h, \pi}}\left[y_{i+1}^{n, \pi}\left(X_{t_{i+1}}^{h, \pi}\right) \mid X_{t_{i}}^{h, \pi}\right]+f\left(t_{i}, S_{t_{i}}^{h, \pi}, y_{i}^{n-1, \pi}\left(X_{t_{i}}^{h, \pi}\right), z_{i}^{n-1, \pi}\left(X_{t_{i}}^{h, \pi}\right)\right) \Delta_{i} \\
& =y_{i}^{n, \pi}\left(X_{t_{i}}^{h, \pi}\right),
\end{aligned}
$$

where we first use the martingale property of $\Psi_{t_{j}}^{h, \pi, i}$, the fifth equality is due to the induction hypothesis and the sixth one is true because $\left(X_{t_{i}}^{h, \pi}, \mathcal{F}_{t_{i}}\right)$ is Markovian under the measure $Q^{h, \pi}$. Finally, the function $y_{i}^{n, \pi}$ does not depend on $h$, because $\left(X_{t_{i}}^{h, \pi}, \mathcal{F}_{t_{i}}\right)$ has the same transition probability under $Q^{h, \pi}$ as $\left(X_{t_{i}}^{\pi}, \mathcal{F}_{t_{i}}\right)$ has under $P$.

Similarly, we obtain, for $d=1, \ldots, D$,

$$
\begin{aligned}
Z_{d, t_{i}}^{h, n, \pi} & =E\left[\frac{\Delta W_{d, i}^{h, \pi}}{\Delta_{i}}\left(\Psi_{t_{N}}^{h, \pi, i} \phi\left(X_{t_{N}}^{h, \pi}\right)+\sum_{j=i+1}^{N-1} \Psi_{t_{j}}^{h, \pi, i} f\left(t_{j}, S_{t_{j}}^{h, \pi}, Y_{t_{j}}^{h, n-1, \pi}, Z_{t_{j}}^{h, n-1, \pi}\right) \Delta_{j}\right) \mid \mathcal{F}_{t_{i}}\right] \\
& =E\left[\Psi_{t_{N}}^{h, \pi, i} \frac{\Delta W_{d, i}^{h, \pi}}{\Delta_{i}}\left(\phi\left(X_{t_{N}}^{h, \pi}\right)+\sum_{j=i+1}^{N-1} f\left(t_{j}, S_{t_{j}}^{h, \pi}, Y_{t_{j}}^{h, n-1, \pi}, Z_{t_{j}}^{h, n-1, \pi}\right) \Delta_{j}\right) \mid \mathcal{F}_{t_{i}}\right] \\
& =E^{Q^{h, \pi}}\left[\frac{\Delta W_{d, i}^{h, \pi}}{\Delta_{i}} Y_{t_{i+1}}^{h, n, \pi} \mid \mathcal{F}_{t_{i}}\right]=E^{Q^{h, \pi}}\left[\frac{\Delta W_{d, i}^{h, \pi}}{\Delta_{i}} y_{i+1}^{n, \pi}\left(X_{t_{i+1}}^{h, \pi}\right) \mid \mathcal{F}_{t_{i}}\right] \\
& =E^{Q^{h, \pi}}\left[\frac{\Delta W_{d, i}^{h, \pi}}{\Delta_{i}} y_{i+1}^{n, \pi}\left(X_{t_{i+1}}^{h, \pi}\right) \mid X_{t_{i}}^{h, \pi}\right]=z_{d, i}^{n, \pi}\left(X_{t_{i}}^{h, \pi}\right),
\end{aligned}
$$

where we used the independence of $\Delta W_{d, i}^{h, \pi}$ and $X_{t_{i}}^{h, \pi}$ and the notation $z_{i}^{n, \pi}(\cdot)=\left(z_{1, i}^{n, \pi}(\cdot), \ldots, z_{D, i}^{n, \pi}(\cdot)\right)$.

Since the regression functions do not depend on the choice of $h$ and $X_{t_{0}}^{h, \pi}=X_{0}$, we can conclude that the error made by approximating $\left(Y_{0}, Z_{0}\right)$ with $\left(Y_{t_{0}}^{h, n, \pi}, Z_{t_{0}}^{h, n, \pi}\right)$ is independent of $h$. Hence, we can simply choose $h \equiv 0$ for which case the error estimate was already derived in Theorem 2.1.

Corollary 3.2. There are constants $C$ and $C^{*}$ (independent of $h$ ) such that for all $h$

$$
\left|Y_{t_{0}}^{h, n, \pi}-Y_{0}\right|^{2}+\left|Z_{t_{0}}^{h, n, \pi}-Z_{0}\right|^{2} \leq C E\left[\left|X_{T}-X_{t_{N}}^{\pi}\right|^{2}\right]+C|\pi|+C\left(\frac{1}{2}+C^{*}|\pi|\right)^{n}
$$


where $C^{*}$ is the same constant as in Theorem 2.1.

Remark 3.3. Another way to prove this result is to rewrite the iteration scheme under the new measure $Q^{h, \pi}$. Since $\left(S^{h, \pi}, Y^{h, n, \pi}, Z^{h, n, \pi}\right)$ has the same law under the new measure as $\left(S^{\pi}, Y^{n, \pi}, Z^{n, \pi}\right)$ has under $P$ we can derive the above error estimate.

We now add a further assumption which guarantees that $\Psi_{t_{i}}^{h, \pi, 0} Y_{t_{i}}^{h, n, \pi}$ and $\Psi_{t_{i}}^{h, \pi, 0} Z_{t_{i}}^{h, n, \pi}$ are squareintegrable under $P$. This assumption turns out to be essential in order to avoid infinite variances within the Monte Carlo implementation.

A 7. For $0 \leq i \leq N-1$

$$
E\left[\left(\Psi_{t_{N}}^{h, \pi, 0} \phi\left(X_{t_{N}}^{h, \pi}\right)+\sum_{j=i}^{N-1} \Psi_{t_{j}}^{h, \pi, 0} f\left(t_{j}, S_{t_{j}}^{h, \pi}, 0,0\right) \Delta_{j}\right)^{2}\right]<\infty
$$

For the first level of the Picard-iteration the above claim is now straightforward:

Lemma 3.4. It holds that $\left(\Psi_{t_{i}}^{h, \pi, 0} Y_{t_{i}}^{h, 1, \pi}, \Psi_{t_{i}}^{h, \pi, 0} Z_{t_{i}}^{h, 1, \pi}\right) \in L^{2}(P)$ for every $0 \leq i \leq N$.

Proof. Since $\Psi_{t_{j}}^{h, \pi, i}=\Psi_{t_{j}}^{h, \pi, 0} / \Psi_{t_{i}}^{h, \pi, 0}$ and $\Psi_{t_{i}}^{h, \pi, 0}$ is $\mathcal{F}_{t_{i}}$-measurable we obtain for $0 \leq i \leq N$ :

$$
\begin{aligned}
& \Psi_{t_{i}}^{h, \pi, 0} Y_{t_{i}}^{h, n, \pi}=E\left[\Psi_{t_{N}}^{h, \pi, 0} \phi\left(X_{t_{N}}^{h, \pi}\right)+\sum_{j=i}^{N-1} \Psi_{t_{j}}^{h, \pi, 0} f\left(t_{j}, S_{t_{j}}^{h, \pi}, Y_{t_{j}}^{h, n-1, \pi}, Z_{t_{j}}^{h, n-1, \pi}\right) \Delta_{j} \mid \mathcal{F}_{t_{i}}\right] \\
& \Psi_{t_{i}}^{h, \pi, 0} Z_{d, t_{i}}^{h, n, \pi}=E\left[\frac{\Delta W_{d, i}^{h, \pi}}{\Delta_{i}}\left(\Psi_{t_{N}}^{h, \pi, 0} \phi\left(X_{t_{N}}^{h, \pi}\right)+\sum_{j=i+1}^{N-1} \Psi_{t_{j}}^{h, \pi, 0} f\left(t_{j}, S_{t_{j}}^{h, \pi}, Y_{t_{j}}^{h, n-1, \pi}, Z_{t_{j}}^{h, n-1, \pi}\right) \Delta_{j}\right) \mid \mathcal{F}_{t_{i}}\right] .
\end{aligned}
$$

Consequently for $n=1$

$$
E\left[\left|\Psi_{t_{i}}^{h, \pi, 0} Y_{t_{i}}^{h, 1, \pi}\right|^{2}\right] \leq E\left[\left(\Psi_{t_{N}}^{h, \pi, 0} \phi\left(X_{t_{N}}^{h, \pi}\right)+\sum_{j=i}^{N-1} \Psi_{t_{j}}^{h, \pi, 0} f\left(t_{j}, S_{t_{j}}^{h, \pi}, 0,0\right) \Delta_{j}\right)^{2}\right]<\infty
$$

and by Hölder's inequality

$$
\begin{aligned}
E\left[\left|\Psi_{t_{i}}^{h, \pi, 0} Z_{d, t_{i}}^{h, 1, \pi}\right|^{2}\right] & \leq E\left[\frac{\left(\Delta W_{d, i}^{h, \pi}\right)^{2}}{\Delta_{i}^{2}}\right] E\left[\left(\Psi_{t_{N}}^{h, \pi, 0} \phi\left(X_{t_{N}}^{h, \pi}\right)+\sum_{j=i+1}^{N-1} \Psi_{t_{j}}^{h, \pi, 0} f\left(t_{j}, S_{t_{j}}^{h, \pi}, 0,0\right) \Delta_{j}\right)^{2}\right] \\
& \leq\left(\frac{2}{\Delta_{i}}+2 C_{h}^{2}\right) E\left[\left(\Psi_{t_{N}}^{h, \pi, 0} \phi\left(X_{t_{N}}^{h, \pi}\right)+\sum_{j=i+1}^{N-1} \Psi_{t_{j}}^{h, \pi, 0} f\left(t_{j}, S_{t_{j}}^{h, \pi}, 0,0\right) \Delta_{j}\right)^{2}\right]<\infty
\end{aligned}
$$

In order to derive the analogue result for $n>1$ we now state some a priori estimates generalizing Lemma 7 in [4].

Lemma 3.5. Suppose $\Gamma$ and $\gamma$ are positive real numbers, $y^{\iota}, z^{\iota}, \iota=1,2$ are adapted processes and

$$
\begin{aligned}
\Psi_{t_{i}}^{h, \pi, 0} Y_{t_{i}}^{(\iota)} & =E\left[\Psi_{t_{N}}^{h, \pi, 0} \phi\left(X_{t_{N}}^{h, \pi}\right)+\sum_{j=i}^{N-1} \Psi_{t_{j}}^{h, \pi, 0} f\left(t_{j}, S_{t_{j}}^{h, \pi}, y_{t_{j}}^{(\iota)}, z_{t_{j}}^{(\iota)}\right) \Delta_{j} \mid \mathcal{F}_{t_{i}}\right] \\
\Psi_{t_{i}}^{h, \pi, 0} Z_{d, t_{i}}^{(\iota)} & =E\left[\frac{\Delta W_{d, i}^{h, \pi}}{\Delta_{i}}\left(\Psi_{t_{N}}^{h, \pi, 0} \phi\left(X_{t_{N}}^{h, \pi}\right)+\sum_{j=i+1}^{N-1} \Psi_{t_{j}}^{h, \pi, 0} f\left(t_{j}, S_{t_{j}}^{h, \pi}, y_{t_{j}}^{(\iota)}, z_{t_{j}}^{(\iota)}\right) \Delta_{j}\right) \mid \mathcal{F}_{t_{i}}\right] .
\end{aligned}
$$


Then

$$
\begin{aligned}
& \max _{0 \leq i \leq N} \lambda_{i} E\left[\left|\Psi_{t_{i}}^{h, \pi, 0} Y_{t_{i}}^{(1)}-\Psi_{t_{i}}^{h, \pi, 0} Y_{t_{i}}^{(2)}\right|^{2}\right]+\sum_{i=0}^{N-1} \lambda_{i} E\left[\left|\Psi_{t_{i}}^{h, \pi, 0} Z_{t_{i}}^{(1)}-\Psi_{t_{i}}^{h, \pi, 0} Z_{t_{i}}^{(2)}\right|^{2}\right] \Delta_{i} \\
\leq & K^{2}(T+1)\left(\left(|\pi|+\frac{1}{\Gamma}\right)\left(2 D\left(\gamma+C_{h}^{2}\right) T+1\right)+2 \frac{D}{\gamma}\right) \\
& \times\left(\frac{1}{T} \sum_{i=0}^{N-1} \lambda_{i} E\left[\left|\Psi_{t_{i}}^{h, \pi, 0} y_{t_{i}}^{(1)}-\Psi_{t_{i}}^{h, \pi, 0} y_{t_{i}}^{(2)}\right|^{2}\right] \Delta_{i}+\sum_{i=0}^{N-1} \lambda_{i} E\left[\left|\Psi_{t_{i}}^{h, \pi, 0} z_{t_{i}}^{(1)}-\Psi_{t_{i}}^{h, \pi, 0} z_{t_{i}}^{(2)}\right|^{2}\right] \Delta_{i}\right),
\end{aligned}
$$

where $\lambda_{0}=1$ and $\lambda_{i}=\left(1+\Gamma \Delta_{i-1}\right) \lambda_{i-1}$.

The proof is given in the Appendix.

With this result at hand we can conclude:

Corollary 3.6. For every $0 \leq i \leq N$ and $n \in \mathbb{N}$ we have $\left(\Psi_{t_{i}}^{h, \pi, 0} Y_{t_{i}}^{h, n, \pi}, \Psi_{t_{i}}^{h, \pi, 0} Z_{t_{i}}^{h, n, \pi}\right) \in L^{2}(P)$.

Proof. Considering $\left(Y^{h, n, \pi}, Z^{h, n, \pi}\right)$ and $\left(Y^{h, n-1, \pi}, Z^{h, n-1, \pi}\right)$ we are in the situation of Lemma 3.5 with $y^{(1)}=Y^{h, n-1, \pi}, y^{(2)}=Y^{h, n-2, \pi}, z^{(1)}=Z^{h, n-1, \pi}$ and $z^{(2)}=Z^{h, n-2, \pi}$. Hence, choosing $\gamma=8 D K^{2}(T+1)$ and $\Gamma=4 K^{2}(T+1)\left(2 D\left(\gamma+C_{h}^{2}\right) T+1\right)$ we can estimate

$$
\begin{gathered}
\max _{0 \leq i \leq N} \lambda_{i} E\left[\left|\Psi_{t_{i}}^{h, \pi, 0} Y_{t_{i}}^{h, n, \pi}-\Psi_{t_{i}}^{h, \pi, 0} Y_{t_{i}}^{h, n-1, \pi}\right|^{2}\right]+\sum_{i=0}^{N-1} \lambda_{i} E\left[\left|\Psi_{t_{i}}^{h, \pi, 0} Z_{t_{i}}^{h, n, \pi}-\Psi_{t_{i}}^{h, \pi, 0} Z_{t_{i}}^{h, n-1, \pi}\right|^{2}\right] \Delta_{i} \\
\leq\left(\frac{\Gamma}{4}|\pi|+\frac{1}{2}\right)\left(\max _{0 \leq i \leq N} \lambda_{i} E\left[\left|\Psi_{t_{i}}^{h, \pi, 0} Y_{t_{i}}^{h, n-1, \pi}-\Psi_{t_{i}}^{h, \pi, 0} Y_{t_{i}}^{h, n-2, \pi}\right|^{2}\right]\right. \\
\left.+\sum_{i=0}^{N-1} \lambda_{i} E\left[\left|\Psi_{t_{i}}^{h, \pi, 0} Z_{t_{i}}^{h, n-1, \pi}-\Psi_{t_{i}}^{h, \pi, 0} Z_{t_{i}}^{h, n-2, \pi}\right|^{2}\right] \Delta_{i}\right) \\
\leq\left(\frac{\Gamma}{4}|\pi|+\frac{1}{2}\right)^{n-1}\left(\max _{0 \leq i \leq N} \lambda_{i} E\left[\left|\Psi_{t_{i}}^{h, \pi, 0} Y_{t_{i}}^{h, 1, \pi}\right|^{2}\right]+\sum_{i=0}^{N-1} \lambda_{i} E\left[\left|\Psi_{t_{i}}^{h, \pi, 0} Z_{t_{i}}^{h, 1, \pi}\right|^{2}\right] \Delta_{i}\right) \\
\leq e^{\Gamma T}\left(\frac{\Gamma}{4}|\pi|+\frac{1}{2}\right)^{n-1}\left(\max _{0 \leq i \leq N} E\left[\left|\Psi_{t_{i}}^{h, \pi, 0} Y_{t_{i}}^{h, 1, \pi}\right|^{2}\right]+\sum_{i=0}^{N-1} E\left[\left|\Psi_{t_{i}}^{h, \pi, 0} Z_{t_{i}}^{h, 1, \pi}\right|^{2}\right] \Delta_{i}\right)<\infty .
\end{gathered}
$$

Here we iteratively applied Lemma 3.5 and the last estimate is due to Lemma 3.4.

The claim now follows by induction. For $n=1$ it is true by Lemma 3.4. Now, suppose it is valid for some $(n-1) \in \mathbb{N}$, then

$$
E\left[\left|\Psi_{t_{i}}^{h, \pi, 0} Y_{t_{i}}^{h, n, \pi}\right|^{2}\right] \leq 2 E\left[\left|\Psi_{t_{i}}^{h, \pi, 0} Y_{t_{i}}^{h, n-1, \pi}\right|^{2}\right]+2 E\left[\left|\Psi_{t_{i}}^{h, \pi, 0} Y_{t_{i}}^{h, n, \pi}-\Psi_{t_{i}}^{h, \pi, 0} Y_{t_{i}}^{h, n-1, \pi}\right|^{2}\right] .
$$

The first term is finite by the induction hypothesis, the second one can be estimated with the above calculation. For the $Z$-part we can proceed analogously.

\section{Least-squares Monte Carlo}

To get a fully implementable algorithm we have to approximate the conditional expectations by some estimator. In this section we describe a simulation based least-squares Monte Carlo estimator and prove its convergence. Recall that the least-squares method can be applied to estimate the conditional expectation of a square-integrable random variable, see e.g. [9, 20]. However, we cannot guarantee that the processes 
$\left(Y^{h, n, \pi}, Z^{h, n, \pi}\right)$ are square integrable in general under the measure $P$. Therefore we cannot apply the least-squares approach directly to $\left(Y^{h, n, \pi}, Z^{h, n, \pi}\right)$, but work with $\left(\Psi^{h, \pi, 0} Y^{h, n, \pi}, \Psi^{h, \pi, 0} Z^{h, n, \pi}\right)$ instead.

As explained above, our remaining task is to estimate

$$
\begin{aligned}
Y_{t_{i}}^{h, n, \pi} & =E\left[\Psi_{t_{N}}^{h, \pi, i} \phi\left(X_{t_{N}}^{h, \pi}\right)+\sum_{j=i}^{N-1} \Psi_{t_{j}}^{h, \pi, i} f\left(t_{j}, S_{t_{j}}^{h, \pi}, Y_{t_{j}}^{h, n-1, \pi}, Z_{t_{j}}^{h, n-1, \pi}\right) \Delta_{j} \mid \mathcal{F}_{t_{i}}\right], \\
Z_{d, t_{i}}^{h, n} & =E\left[\frac{\Delta W_{d, i}^{h, \pi}}{\Delta_{i}}\left(\Psi_{t_{N}}^{h, \pi, i} \phi\left(X_{t_{N}}^{h, \pi}\right)+\sum_{j=i+1}^{N-1} \Psi_{t_{j}}^{h, \pi, i} f\left(t_{j}, S_{t_{j}}^{h, \pi}, Y_{t_{j}}^{h, n-1, \pi}, Z_{t_{j}}^{h, n-1, \pi}\right) \Delta_{j}\right) \mid \mathcal{F}_{t_{i}}\right],
\end{aligned}
$$

which we will do in the sequel. For any random variable $V$ such that $\Psi_{t_{i}}^{h, \pi, 0} V \in L^{2}\left(\mathcal{F}_{t_{N}}, P\right)$ and $E\left[V \mid \mathcal{F}_{t_{i}}\right]=$ $E\left[V \mid X_{t_{i}}^{h, \pi}\right]$, we write $E\left[V \mid \mathcal{F}_{t_{i}}\right]=\left(\Psi_{t_{i}}^{h, \pi, 0}\right)^{-1} E\left[\Psi_{t_{i}}^{h, \pi, 0} V \mid \mathcal{F}_{t_{i}}\right]$ and note that

$$
E\left[\Psi_{t_{i}}^{h, \pi, 0} V \mid \mathcal{F}_{t_{i}}\right]=\Psi_{t_{i}}^{h, \pi, 0} E\left[V \mid X_{t_{i}}^{h, \pi}\right] .
$$

Consequently, $E\left[\Psi_{t_{i}}^{h, \pi, 0} V \mid \mathcal{F}_{t_{i}}\right]$ is the orthogonal projection on the space $L^{2}\left(\mathcal{G}_{i}^{h, \pi}, P\right)$, where $\mathcal{G}_{i}^{h, \pi}$ denotes the $\sigma$-field generated by the random variables of the form $\Psi_{t_{i}}^{h, \pi, 0} v\left(X_{t_{i}}^{h, \pi}\right)$ for deterministic and measurable functions $v$. We now replace this projection by a projection on a finite dimensional subspace. To do so, we choose, for each time partition point, $D+1$ sets of basis functions

$$
\begin{aligned}
& \left\{p_{0, i, 1}(\cdot), \ldots, p_{0, i, K_{0, i}}(\cdot)\right\} \text { for the estimation of } Y_{t_{i}}^{h, n, \pi} \text { and } \\
& \left\{p_{d, i, 1}(\cdot), \ldots, p_{d, i, K_{d, i}}(\cdot)\right\} \text { for the estimation of } Z_{d, t_{i}}^{h, n} .
\end{aligned}
$$

We assume that

$$
\eta_{d, i, k}^{h}:=\Psi_{t_{i}}^{h, \pi, 0} p_{d, i, k}\left(X_{t_{i}}^{h, \pi}\right)
$$

satisfy $E\left[\left|\eta_{d, i, k}^{h}\right|^{2}\right]<\infty$ for every $0 \leq d \leq D, 0 \leq i \leq N-1$ and $0 \leq k \leq K_{d, i}$, and that $\left(\eta_{d, i, 1}^{h}, \ldots, \eta_{d, i, K_{d, i}}^{h}\right)$ are linearly independent for every $0 \leq d \leq D, 0 \leq i \leq N-1$. Now we define $\Lambda_{d, i}^{h}=\operatorname{span}\left(\eta_{d, i, k}^{h}\right)$ and denote by $P_{d, i}^{h}$ the orthogonal (in the $L^{2}$-sense) projection on $\Lambda_{d, i}^{h}$. As these spaces are finite dimensional, there are coefficients $\alpha_{d, i, k}(V)$ such that

$$
P_{d, i}^{h}\left[\Psi_{t_{i}}^{h, \pi, 0} V\right]=\sum_{k=1}^{K_{d, i}} \alpha_{d, i, k}(V) \Psi_{t_{i}}^{h, \pi, 0} p_{d, i, k}\left(X_{t_{i}}^{h, \pi}\right) .
$$

The inner-product matrices associated to the chosen bases are

$$
\mathcal{B}_{d, i}^{h}=E\left[\eta_{d, i, k}^{h} \eta_{d, i, l}^{h}\right]_{k, l=1, \ldots, K_{d, i}}
$$

Hence we obtain as coefficients

$$
\alpha_{d, i}(V)=\left(\mathcal{B}_{d, i}^{h}\right)^{-1} E\left[\eta_{d, i}^{h} V\right]
$$

where $\eta_{d, i}^{h}=\left(\eta_{d, i, 1}^{h}, \ldots, \eta_{d, i, K_{d, i}}^{h}\right)^{\top}$ and $\alpha_{d, i}(V)=\left(\alpha_{d, i, 1}(V), \ldots, \alpha_{d, i, K_{d, i}}(V)\right)^{\top}$. Finally, the corresponding estimator for $E\left[V \mid \mathcal{F}_{t_{i}}\right]=E\left[V \mid X_{t_{i}}^{h, \pi}\right]$, given the basis $\left\{p_{d, i, 1}(\cdot), \ldots, p_{d, i, K_{d, i}}(\cdot)\right\}$, is

$$
\sum_{k=1}^{K_{d, i}} \alpha_{d, i, k}(V) p_{d, i, k}\left(X_{t_{i}}^{h, \pi}\right)
$$


Thanks to Theorem 3.1 and Corollary 3.6 we can apply this machinery for estimating $Y_{t_{i}}^{h, n, \pi}$ and $Z_{d, t_{i}}^{h, \pi}$. As estimators for these quantities we define

$$
\begin{aligned}
\widehat{Y}_{t_{i}}^{h, n, \pi} & =\left(\Psi_{t_{i}}^{h, \pi, 0}\right)^{-1} P_{0, i}^{h}\left[\Psi_{t_{N}}^{h, \pi, 0} \phi\left(X_{t_{N}}^{h, \pi}\right)+\sum_{j=i}^{N-1} \Psi_{t_{j}}^{h, \pi, 0} f\left(t_{j}, S_{t_{j}}^{h, \pi}, \widehat{Y}_{t_{j}}^{h, n-1, \pi}, \widehat{Z}_{t_{j}}^{h, n-1, \pi}\right) \Delta_{j}\right] \\
& =\sum_{k=1}^{K_{0, i}} \alpha_{0, i, k}^{h, n, \pi} p_{0, i, k}\left(X_{t_{i}}^{h, \pi}\right), \\
\widehat{Z}_{d, t_{i}}^{h, n, \pi} & =\left(\Psi_{t_{i}}^{h, \pi, 0}\right)^{-1} P_{d, i}^{h}\left[\frac{\Delta W_{d, i}^{h, \pi}}{\Delta_{i}}\left(\Psi_{t_{N}}^{h, \pi, 0} \phi\left(X_{t_{N}}^{h, \pi}\right)+\sum_{j=i+1}^{N-1} \Psi_{t_{j}}^{h, \pi, 0} f\left(t_{j}, S_{t_{j}}^{h, \pi}, \widehat{Y}_{t_{j}}^{h, n-1, \pi}, \widehat{Z}_{t_{j}}^{h, n-1, \pi}\right) \Delta_{j}\right)\right] \\
& =\sum_{k=1}^{K_{d, i}} \alpha_{d, i, k}^{h, n, \pi} p_{d, i, k}\left(X_{t_{i}}^{h, \pi}\right)
\end{aligned}
$$

where

$$
\alpha_{0, i}^{h, n, \pi}=\left(\mathcal{B}_{0, i}^{h}\right)^{-1} E\left[\eta_{0, i}^{h}\left(\Psi_{t_{N}}^{h, \pi, 0} \phi\left(X_{t_{N}}^{h, \pi}\right)+\sum_{j=i}^{N-1} \Psi_{t_{j}}^{h, \pi, 0} f\left(t_{j}, S_{t_{j}}^{h, \pi}, \widehat{Y}_{t_{j}}^{h, n-1, \pi}, \widehat{Z}_{t_{j}}^{h, n-1, \pi}\right) \Delta_{j}\right)\right],
$$

and for $d \geq 1$

$$
\begin{aligned}
\alpha_{d, i}^{h, n, \pi}=\left(\mathcal{B}_{d, i}^{h}\right)^{-1} E\left[\eta _ { d , i } ^ { h } \left(\frac { \Delta W _ { d , i } ^ { h , \pi } } { \Delta _ { i } } \left(\Psi_{t_{N}}^{h, \pi, 0} \phi\left(X_{t_{N}}^{h, \pi}\right)\right.\right.\right. & \\
& \left.\left.\left.+\sum_{j=i+1}^{N-1} \Psi_{t_{j}}^{h, \pi, 0} f\left(t_{j}, S_{t_{j}}^{h, \pi}, \widehat{Y}_{t_{j}}^{h, n-1, \pi}, \widehat{Z}_{t_{j}}^{h, n-1, \pi}\right) \Delta_{j}\right)\right)\right],
\end{aligned}
$$

initialized at $\left(\widehat{Y}^{h, 0, \pi}, \widehat{Z}^{h, 0, \pi}\right)=0$.

Remark 4.1. Note that Assumption $A 7$ and Theorem 4.2 below guarantee that the weights in (6)-(7) are finite.

In the following, we analyze the error resulting from the approximation of $\left(\Psi_{t_{i}}^{h, \pi, 0} Y_{t_{i}}^{h, n, \pi}, \Psi_{t_{i}}^{h, \pi, 0} Z_{t_{i}}^{h, n, \pi}\right)$ with $\left(\Psi_{t_{i}}^{h, \pi, 0} \widehat{Y}_{t_{i}}^{h, n, \pi}, \Psi_{t_{i}}^{h, \pi, 0} \widehat{Z}_{t_{i}}^{h, n, \pi}\right)$. Analogously to Bender and Denk [4] this will be done in terms of the projection errors $\left|\Psi_{t_{i}}^{h, \pi, 0} Y_{t_{i}}^{h, n, \pi}-P_{0, i}^{h}\left(\Psi_{t_{i}}^{h, \pi, 0} Y_{t_{i}}^{h, n, \pi}\right)\right|$ and $\left|\Psi_{t_{i}}^{h, \pi, 0} Z_{d, t_{i}}^{h, n, \pi}-P_{d, i}^{h}\left(\Psi_{t_{i}}^{h, \pi, 0} Z_{d, t_{i}}^{h, n, \pi}\right)\right|$. We extend their Theorem 11 (which corresponds to the case $h=0$ ), reflecting the advantage of the Picard-type scheme: The error induced by the approximation of the conditional expectations does neither explode when the number of time steps tends to infinity nor does it blow up if the number of iterations grows. We simply obtain, that the $L^{2}$-error is bounded by a constant times the worst $L^{2}$-projection error occurring during iterations.

Theorem 4.2. There is a constant $C$ depending on the data and the bound of $h$ such that

$$
\begin{gathered}
\max _{0 \leq i \leq N} E\left[\left|\Psi_{t_{i}}^{h, \pi, 0} \widehat{Y}_{t_{i}}^{h, n, \pi}-\Psi_{t_{i}}^{h, \pi, 0} Y_{t_{i}}^{h, n, \pi}\right|^{2}\right]+\sum_{i=0}^{N-1} E\left[\left|\Psi_{t_{i}}^{h, \pi, 0} \widehat{Z}_{t_{i}}^{h, n, \pi}-\Psi_{t_{i}}^{h, \pi, 0} Z_{t_{i}}^{h, n, \pi}\right|^{2}\right] \Delta_{i} \\
\leq C\left(\operatorname { m a x } _ { 1 \leq \nu \leq n } \operatorname { m a x } _ { 0 \leq i \leq N } \left(E\left[\left|\Psi_{t_{i}}^{h, \pi, 0} Y_{t_{i}}^{h, \nu, \pi}-P_{0, i}^{h}\left[\Psi_{t_{i}}^{h, \pi, 0} Y_{t_{i}}^{h, \nu, \pi}\right]\right|^{2}\right]\right.\right. \\
\left.\left.+\sum_{d=1}^{D} E\left[\left|\Psi_{t_{i}}^{h, \pi, 0} Z_{d, t_{i}}^{h, \nu, \pi}-P_{d, i}^{h}\left[\Psi_{t_{i}}^{h, \pi, 0} Z_{d, t_{i}}^{h, \nu, \pi}\right]\right|^{2}\right]\right)\right)
\end{gathered}
$$

for sufficiently small $|\pi|$. 
Proof. Define

$$
\begin{aligned}
\bar{Y}_{t_{i}}^{h, n, \pi}= & \left(\Psi_{t_{i}}^{h, \pi, 0}\right)^{-1} E\left[\Psi_{t_{N}}^{h, \pi, 0} \phi\left(X_{t_{N}}^{h, \pi}\right)+\sum_{j=i}^{N-1} \Psi_{t_{j}}^{h, \pi, 0} f\left(t_{j}, S_{t_{j}}^{h, \pi}, \widehat{Y}_{t_{j}}^{h, n-1, \pi}, \widehat{Z}_{t_{j}}^{h, n-1, \pi}\right) \Delta_{j} \mid \mathcal{F}_{t_{i}}\right], \\
\bar{Z}_{d, t_{i}}^{h, n, \pi}= & \left(\Psi_{t_{i}}^{h, \pi, 0}\right)^{-1} E\left[\frac { \Delta W _ { d , i } ^ { h , \pi } } { \Delta _ { i } } \left(\Psi_{t_{N}}^{h, \pi, 0} \phi\left(X_{t_{N}}^{h, \pi}\right)\right.\right. \\
& \left.\left.+\sum_{j=i+1}^{N-1} \Psi_{t_{j}}^{h, \pi, 0} f\left(t_{j}, S_{t_{j}}^{h, \pi}, \widehat{Y}_{t_{j}}^{h, n-1, \pi}, \widehat{Z}_{t_{j}}^{h, n-1, \pi}\right) \Delta_{j}\right) \mid \mathcal{F}_{t_{i}}\right] .
\end{aligned}
$$

Then we obtain

$$
\begin{aligned}
& P_{0, i}^{h}\left[\Psi_{t_{i}}^{h, \pi, 0} \bar{Y}_{t_{i}}^{h, n, \pi}-\Psi_{t_{i}}^{h, \pi, 0} Y_{t_{i}}^{h, n, \pi}\right]=\Psi_{t_{i}}^{h, \pi, 0} \widehat{Y}_{t_{i}}^{h, n, \pi}-P_{0, i}^{h}\left[\Psi_{t_{i}}^{h, \pi, 0} Y_{t_{i}}^{h, n, \pi}\right], \\
& P_{d, i}^{h}\left[\Psi_{t_{i}}^{h, \pi, 0} \bar{Z}_{d, t_{i}}^{h, n, \pi}-\Psi_{t_{i}}^{h, \pi, 0} Z_{d, t_{i}}^{h, n}\right]=\Psi_{t_{i}}^{h, \pi, 0} \widehat{Z}_{d, t_{i}}^{h, n, \pi}-P_{d, i}^{h}\left[\Psi_{t_{i}}^{h, \pi, 0} Z_{d, t_{i}}^{h, n, \pi}\right] .
\end{aligned}
$$

Due to the orthogonality of the projection we also have

$$
\begin{aligned}
E\left[\left|\Psi_{t_{i}}^{h, \pi, 0} \widehat{Y}_{t_{i}}^{h, n, \pi}-\Psi_{t_{i}}^{h, \pi, 0} Y_{t_{i}}^{h, n, \pi}\right|^{2}\right]= & E\left[\left|\Psi_{t_{i}}^{h, \pi, 0} \widehat{Y}_{t_{i}}^{h, n, \pi}-P_{0, i}^{h}\left[\Psi_{t_{i}}^{h, \pi, 0} Y_{t_{i}}^{h, n, \pi}\right]\right|^{2}\right] \\
& +E\left[\left|P_{0, i}^{h}\left[\Psi_{t_{i}}^{h, \pi, 0} Y_{t_{i}}^{h, n, \pi}\right]-\Psi_{t_{i}}^{h, \pi, 0} Y_{t_{i}}^{h, n, \pi}\right|^{2}\right],
\end{aligned}
$$

and the analogous equation holds for $Z_{d, t_{i}}^{h, n}$. Consequently, we get for any $0 \leq i \leq N$,

$$
\begin{aligned}
& E\left[\left|\Psi_{t_{i}}^{h, \pi, 0} \widehat{Y}_{t_{i}}^{h, n, \pi}-\Psi_{t_{i}}^{h, \pi, 0} Y_{t_{i}}^{h, n, \pi}\right|^{2}\right] \\
= & E\left[\left|\Psi_{t_{i}}^{h, \pi, 0} \widehat{Y}_{t_{i}}^{h, n, \pi}-P_{0, i}^{h}\left[\Psi_{t_{i}}^{h, \pi, 0} Y_{t_{i}}^{h, n, \pi}\right]\right|^{2}\right]+E\left[\left|P_{0, i}^{h}\left[\Psi_{t_{i}}^{h, \pi, 0} Y_{t_{i}}^{h, n, \pi}\right]-\Psi_{t_{i}}^{h, \pi, 0} Y_{t_{i}}^{h, n, \pi}\right|^{2}\right] \\
= & E\left[\left|P_{0, i}^{h}\left[\Psi_{t_{i}}^{h, \pi} \bar{Y}_{t_{i}}^{h, n, \pi}-\Psi_{t_{i}}^{h, \pi, 0} Y_{t_{i}}^{h, n, \pi}\right]\right|^{2}\right]+E\left[\left|P_{0, i}^{h}\left[\Psi_{t_{i}}^{h, \pi, 0} Y_{t_{i}}^{h, n, \pi}\right]-\Psi_{t_{i}}^{h, \pi, 0} Y_{t_{i}}^{h, n, \pi}\right|^{2}\right] \\
\leq & E\left[\left|\Psi_{t_{i}}^{h, \pi, 0} \bar{Y}_{t_{i}}^{h, n, \pi}-\Psi_{t_{i}}^{h, \pi, 0} Y_{t_{i}}^{h, n, \pi}\right|^{2}\right]+E\left[\left|P_{0, i}^{h}\left[\Psi_{t_{i}}^{h, \pi, 0} Y_{t_{i}}^{h, n, \pi}\right]-\Psi_{t_{i}}^{h, \pi, 0} Y_{t_{i}}^{h, n, \pi}\right|^{2}\right],
\end{aligned}
$$

where we used in the last step that nontrivial orthogonal projections have norm 1 . In the same way we get

$$
\begin{aligned}
& E\left[\left|\Psi_{t_{i}}^{h, \pi, 0} \widehat{Z}_{t_{i}}^{h, n, \pi}-\Psi_{t_{i}}^{h, \pi, 0} Z_{t_{i}}^{h, n, \pi}\right|^{2}\right] \\
\leq & \sum_{d=1}^{D} E\left[\left|\Psi_{t_{i}}^{h, \pi, 0} \bar{Z}_{d, t_{i}}^{h, n, \pi}-\Psi_{t_{i}}^{h, \pi, 0} Z_{d, t_{i}}^{h, n, \pi}\right|^{2}\right]+\sum_{d=1}^{D} E\left[\left|P_{d, i}^{h}\left[\Psi_{t_{i}}^{h, \pi, 0} Z_{d, t_{i}}^{h, n, \pi}\right]-\Psi_{t_{i}}^{h, \pi, 0} Z_{d, t_{i}}^{h, n, \pi}\right|^{2}\right] .
\end{aligned}
$$

Multiplying these inequalities with the weights $\lambda_{i}$, satisfying $\lambda_{0}=1$ and $\lambda_{i}=\left(1+\Gamma \Delta_{i-1}\right) \lambda_{i-1}$ for some $\Gamma>0$ to be specified later, we obtain

$$
\begin{aligned}
& \max _{0 \leq i \leq N} \lambda_{i} E\left[\left|\Psi_{t_{i}}^{h, \pi, 0} \widehat{Y}_{t_{i}}^{h, n, \pi}-\Psi_{t_{i}}^{h, \pi, 0} Y_{t_{i}}^{h, n, \pi}\right|^{2}\right]+\sum_{i=0}^{N-1} \lambda_{i} E\left[\left|\Psi_{t_{i}}^{h, \pi, 0} \widehat{Z}_{t_{i}}^{h, n, \pi}-\Psi_{t_{i}}^{h, \pi, 0} Z_{t_{i}}^{h, n, \pi}\right|^{2}\right] \Delta_{i} \\
\leq & \max _{0 \leq i \leq N} \lambda_{i} E\left[\left|\Psi_{t_{i}}^{h, \pi, 0} \bar{Y}_{t_{i}}^{h, n, \pi}-\Psi_{t_{i}}^{h, \pi, 0} Y_{t_{i}}^{h, n, \pi}\right|^{2}\right]+\sum_{i=0}^{N-1} \lambda_{i} E\left[\left|\Psi_{t_{i}}^{h, \pi, 0} \bar{Z}_{t_{i}}^{h, n, \pi}-\Psi_{t_{i}}^{h, \pi, 0} Z_{t_{i}}^{h, n, \pi}\right|^{2}\right] \Delta_{i} \\
& +\max _{0 \leq i \leq N} \lambda_{i} E\left[\left|P_{0, i}^{h}\left[\Psi_{t_{i}}^{h, \pi, 0} Y_{t_{i}}^{h, n, \pi}\right]-\Psi_{t_{i}}^{h, \pi, 0} Y_{t_{i}}^{h, n, \pi}\right|^{2}\right] \\
& +\sum_{d=1}^{D} \sum_{i=0}^{N-1} \lambda_{i} E\left[\left|P_{d, i}^{h}\left[\Psi_{t_{i}}^{h, \pi, 0} Z_{d, t_{i}}^{h, n, \pi}\right]-\Psi_{t_{i}}^{h, \pi, 0} Z_{d, t_{i}}^{h, n, \pi}\right|^{2}\right] \Delta_{i} .
\end{aligned}
$$


Applying Lemma 3.5 with $\gamma=8 D K^{2}(T+1)$ and $\Gamma=4 K^{2}(T+1)\left(2 D\left(\gamma+C_{h}^{2}\right) T+1\right)$ yields

$$
\begin{gathered}
\max _{0 \leq i \leq N} \lambda_{i} E\left[\left|\Psi_{t_{i}}^{h, \pi, 0} \bar{Y}_{t_{i}}^{h, n, \pi}-\Psi_{t_{i}}^{h, \pi, 0} Y_{t_{i}}^{h, n, \pi}\right|^{2}\right]+\sum_{i=0}^{N-1} \lambda_{i} E\left[\left|\Psi_{t_{i}}^{h, \pi, 0} \bar{Z}_{t_{i}}^{h, n, \pi}-\Psi_{t_{i}}^{h, \pi, 0} Z_{t_{i}}^{h, n, \pi}\right|^{2}\right] \Delta_{i} \\
\leq\left(\frac{\Gamma}{4}|\pi|+\frac{1}{2}\right)\left(\max _{0 \leq i \leq N} \lambda_{i} E\left[\left|\Psi_{t_{i}}^{h, \pi} \widehat{Y}_{t_{i}}^{h, n-1, \pi}-\Psi_{t_{i}}^{h, \pi, 0} Y_{t_{i}}^{h, n-1, \pi}\right|^{2}\right]\right. \\
\left.+\sum_{i=0}^{N-1} \lambda_{i} E\left[\left|\Psi_{t_{i}}^{h, \pi, 0} \widehat{Z}_{t_{i}}^{h, n-1, \pi}-\Psi_{t_{i}}^{h, \pi, 0} Z_{t_{i}}^{h, n-1, \pi}\right|^{2}\right] \Delta_{i}\right) .
\end{gathered}
$$

Putting estimates (8)-(9) together we obtain

$$
\begin{gathered}
\max _{0 \leq i \leq N} \lambda_{i} E\left[\left|\Psi_{t_{i}}^{h, \pi, 0} \widehat{Y}_{t_{i}}^{h, n, \pi}-\Psi_{t_{i}}^{h, \pi, 0} Y_{t_{i}}^{h, n, \pi}\right|^{2}\right]+\sum_{i=0}^{N-1} \lambda_{i} E\left[\left|\Psi_{t_{i}}^{h, \pi, 0} \widehat{Z}_{t_{i}}^{h, n, \pi}-\Psi_{t_{i}}^{h, \pi, 0} Z_{t_{i}}^{h, n, \pi}\right|^{2}\right] \Delta_{i} \\
\leq \max _{0 \leq i \leq N} \lambda_{i} E\left[\left|P_{0, i}^{h}\left[\Psi_{t_{i}}^{h, \pi, 0} Y_{t_{i}}^{h, n, \pi}\right]-\Psi_{t_{i}}^{h, \pi, 0} Y_{t_{i}}^{h, n, \pi}\right|^{2}\right] \\
+\sum_{d=1}^{D} \sum_{i=0}^{N-1} \lambda_{i} E\left[\left|P_{d, i}^{h}\left[\Psi_{t_{i}}^{h, \pi, 0} Z_{d, t_{i}}^{h, n, \pi}\right]-\Psi_{t_{i}}^{h, \pi, 0} Z_{d, t_{i}}^{h, n, \pi}\right|^{2}\right] \Delta_{i} \\
+\left(\frac{\Gamma}{4}|\pi|+\frac{1}{2}\right)\left(\max _{0 \leq i \leq N} \lambda_{i} E\left[\left|\Psi_{t_{i}}^{h, \pi, 0} \widehat{Y}_{t_{i}}^{h, n-1, \pi}-\Psi_{t_{i}}^{h, \pi, 0} Y_{t_{i}}^{h, n-1, \pi}\right|^{2}\right]\right. \\
\left.+\sum_{i=0}^{N-1} \lambda_{i} E\left[\left|\Psi_{t_{i}}^{h, \pi, 0} \widehat{Z}_{t_{i}}^{h, n-1, \pi}-\Psi_{t_{i}}^{h, \pi, 0} Z_{t_{i}}^{h, n-1, \pi}\right|^{2}\right] \Delta_{i}\right) .
\end{gathered}
$$

Iterating this inequality yields

$$
\begin{gathered}
\max _{0 \leq i \leq N} E\left[\left|\Psi_{t_{i}}^{h, \pi, 0} \widehat{Y}_{t_{i}}^{h, n, \pi}-\Psi_{t_{i}}^{h, \pi, 0} Y_{t_{i}}^{h, n, \pi}\right|^{2}\right]+\sum_{i=0}^{N-1} E\left[\left|\Psi_{t_{i}}^{h, \pi, 0} \widehat{Z}_{t_{i}}^{h, n, \pi}-\Psi_{t_{i}}^{h, \pi, 0} Z_{t_{i}}^{h, n, \pi}\right|^{2}\right] \Delta_{i} \\
\leq \sum_{\nu=1}^{n}\left(\frac{\Gamma}{4}|\pi|+\frac{1}{2}\right)^{n-\nu}\left(\max _{0 \leq i \leq N} \lambda_{i} E\left[\left|P_{0, i}^{h}\left[\Psi_{t_{i}}^{h, \pi, 0} Y_{t_{i}}^{h, \nu, \pi}\right]-\Psi_{t_{i}}^{h, \pi, 0} Y_{t_{i}}^{h, \nu, \pi}\right|^{2}\right]\right. \\
\left.+\sum_{d=1}^{D} \sum_{i=0}^{N-1} \lambda_{i} E\left[\left|P_{d, i}^{h}\left[\Psi_{t_{i}}^{h, \pi, 0} Z_{d, t_{i}}^{h, \nu, \pi}\right]-\Psi_{t_{i}}^{h, \pi, 0} Z_{d, t_{i}}^{h, \nu, \pi}\right|^{2}\right] \Delta_{i}\right) \\
\leq e^{\Gamma T} \sum_{\nu=1}^{n}\left(\frac{\Gamma}{4}|\pi|+\frac{1}{2}\right)^{n-\nu}\left(\max _{0 \leq i \leq N} E\left[\left|P_{0, i}^{h}\left[\Psi_{t_{i}}^{h, \pi, 0} Y_{t_{i}}^{h, \nu, \pi}\right]-\Psi_{t_{i}}^{h, \pi, 0} Y_{t_{i}}^{h, \nu, \pi}\right|^{2}\right]\right. \\
\left.+\sum_{d=1}^{D} \sum_{i=0}^{N-1} E\left[\left|P_{d, i}^{h}\left[\Psi_{t_{i}}^{h, \pi, 0} Z_{d, t_{i}}^{h, \nu, \pi}\right]-\Psi_{t_{i}}^{h, \pi, 0} Z_{d, t_{i}}^{h, \nu, \pi}\right|^{2}\right] \Delta_{i}\right) .
\end{gathered}
$$

Hence, the claim finally follows if $|\pi|$ is small enough.

Remark 4.3. The proof of the above theorem only made use of the fact that orthogonal projections have norm 1. Hence, it holds for orthogonal projections on any, possibly infinite-dimensional, subspaces $\Lambda_{d, i}^{h} \subset L^{2}\left(\mathcal{G}_{i}^{h, \pi}, P\right)$.

The final approximation step of our algorithm replaces the expectations in (4) and (5) by their simulation-based counterparts. That is, we assume that we have $L \geq \max _{d, i}\left\{K_{d, i}\right\}$ independent draws from $\left(\Delta W_{i}^{h, \pi}, X_{t_{i}}^{h, \pi}, \phi\left(X_{t_{N}}^{h, \pi}\right), \Psi_{t_{i}}^{h, \pi, j}, \eta_{d, i}^{h}\right)$ which we denote by $\left(\Delta_{\lambda} W_{i}^{h, \pi},{ }_{\lambda} X_{t_{i}}^{h, \pi}, \phi\left({ }_{\lambda} X_{t_{N}}^{h, \pi}\right),{ }_{\lambda} \Psi_{t_{i}}^{h, \pi, j},{ }_{\lambda} \eta_{d, i}^{h}\right)$ 
for $\lambda=1, \ldots, L$. The column vectors of these copies are denoted by $\left(\Delta \mathbf{W}_{i}^{h, \pi}, \mathbf{X}_{t_{i}}^{h, \pi}, \phi\left(\mathbf{X}_{t_{N}}^{h, \pi}\right), \mathbf{P s i}_{t_{i}}^{h, \pi, j}, \mathbf{e}_{d, i}^{h}\right)$, e.g.

Define

$$
\mathbf{X}_{t_{i}}^{h, \pi}=\left({ }_{1} X_{t_{i}}^{h, \pi}, \ldots,{ }_{L} X_{t_{i}}^{h, \pi}\right)^{\top} .
$$

$$
\mathcal{A}_{d, i}^{h, L}:=\frac{1}{\sqrt{L}}\left({ }_{\lambda} \eta_{d, i, k}^{h}\right)_{\lambda=1, \ldots, L, k=1, \ldots, K_{d, i}}, d=0, \ldots, D
$$

so that

$$
\mathcal{B}_{d, i}^{h, L}:=\left(\mathcal{A}_{d, i}^{h, L}\right)^{\top} \mathcal{A}_{d, i}^{h, L}=\frac{1}{L}\left(\sum_{\lambda=1}^{L}{ }_{\lambda} \eta_{d, i, k \lambda}^{h} \eta_{d, i, l}^{h}\right)_{k, l=1, \ldots, K_{d, i}} \quad \text { for } d=0, \ldots, D
$$

are the simulation-based analogues to the matrices $\mathcal{B}_{d, i}^{h}$. Since the inverses of $\mathcal{B}_{d, i}^{h, L}$ need not exist, we switch to the pseudo-inverses $\left(\mathcal{A}_{d, i}^{h, L}\right)^{+}$in order to introduce in recursive manner simulation based analogues to (6)-(7) with the help of the least-squares method. In detail we define:

$$
\begin{aligned}
\alpha_{d, i}^{h, 0, \pi, L} & =0, d=0, \ldots, D \\
{ }_{\lambda} \widehat{Y}_{t_{i}}^{h, n-1, \pi} & =\sum_{k=1}^{K_{0, i}}\left({ }_{\lambda} \Psi_{t_{i}}^{h, \pi, 0}\right)^{-1} \alpha_{0, i, k}^{h, n-1, \pi, L}{ }_{\lambda} \eta_{0, i, k}^{h} \\
{ }_{\lambda} \widehat{Z}_{d, t_{i}}^{h, 1, \pi} & =\sum_{k=1}^{K_{d, i}}\left({ }_{\lambda} \Psi_{t_{i}}^{h, \pi, 0}\right)^{-1} \alpha_{d, i, k}^{h, n-1, \pi, L}{ }_{\lambda} \eta_{d, i, k}^{h} \\
\mathbf{f}\left(t_{i}\right) & =\left(f\left(t_{i},{ }_{1} S_{t_{i}}^{h, \pi},{ }_{1} \widehat{Y}_{t_{i}}^{h, n-1, \pi},{ }_{1} \widehat{Z}_{t_{i}}^{h, n-1, \pi}\right), \ldots, f\left(t_{i},{ }_{L} S_{t_{i}}^{h, \pi},{ }_{L} \widehat{Y}_{t_{i}}^{h, n-1, \pi},{ }_{L} \widehat{Z}_{t_{i}}^{h, n-1, \pi}\right)\right)^{\top} \\
\alpha_{0, i}^{h, n, \pi, L} & =\frac{1}{\sqrt{L}}\left(\mathcal{A}_{0, i}^{h, L}\right)^{+}\left(\mathbf{P s i}_{t_{N}}^{h, \pi, 0} \bullet \phi\left(\mathbf{X}_{t_{N}}^{h, \pi}\right)+\sum_{j=i}^{N-1} \mathbf{P s i}_{t_{j}}^{h, \pi, 0} \bullet \mathbf{f}\left(t_{j}\right) \Delta_{j}\right) \\
\alpha_{d, i}^{h, n, \pi, L} & =\frac{1}{\sqrt{L}}\left(\mathcal{A}_{d, i}^{h, L}\right)^{+}\left(\frac{\Delta \mathbf{W}_{d, i}^{h, \pi}}{\Delta_{i}} \bullet\left(\mathbf{P s i}_{t_{N}}^{h, \pi, 0} \bullet \phi\left(\mathbf{X}_{t_{N}}^{h, \pi}\right)+\sum_{j=i+1}^{N-1} \mathbf{P s i}_{t_{j}}^{h, \pi, 0} \bullet \mathbf{f}\left(t_{j}\right) \Delta_{j}\right)\right), d=1, \ldots, D,
\end{aligned}
$$

where $\bullet$ denotes the componentwise multiplication of two vectors and we used the abbreviation $\alpha_{d, i}^{h, n, \pi, L}=$ $\left(\alpha_{d, i, 1}^{h, n, \pi, L}, \ldots, \alpha_{d, i, K_{d, i}}^{h, n, \pi, L}\right)^{\top}$. This enables us to define simulation based estimators by

$$
\begin{aligned}
\widehat{Y}_{t_{i}}^{h, n, \pi, L} & =\sum_{k=1}^{K_{0, i}}\left(\Psi_{t_{i}}^{h, \pi, 0}\right)^{-1} \alpha_{0, i, k}^{h, n, \pi, L} \eta_{0, i, k}^{h}=\sum_{k=1}^{K_{0, i}} \alpha_{0, i, k}^{h, n, \pi, L} p_{0, i, k}\left(X_{t_{i}}^{h, \pi}\right), \\
\widehat{Z}_{d, t_{i}}^{h, n, \pi, L} & =\sum_{k=1}^{K_{d, i}}\left(\Psi_{t_{i}}^{h, \pi, 0}\right)^{-1} \alpha_{d, i, k}^{h, n, \pi, L} \eta_{d, i, k}^{h}=\sum_{k=1}^{K_{d, i}} \alpha_{d, i, k}^{h, n, \pi, L} p_{d, i, k}\left(X_{t_{i}}^{h, \pi}\right), d=1, \ldots, D .
\end{aligned}
$$

Note that the thus constructed coefficients solve linear least-squares problems, e.g.

$$
\begin{aligned}
& \alpha_{0, i}^{h, n, \pi, L}=\underset{\alpha \in \mathbb{R}^{K_{0, i}}}{\operatorname{arginf}} \frac{1}{L} \sum_{\lambda=1}^{L} \mid \alpha^{\top}{ }_{\lambda} \eta_{0, i}^{h}-{ }_{\lambda} \Psi_{t_{N}}^{h, \pi, 0} \phi\left({ }_{\lambda} X_{t_{N}}^{h, \pi}\right) \\
&-\left.\sum_{j=i}^{N-1}{ }_{\lambda} \Psi_{t_{j}}^{h, \pi, 0} f\left(t_{j},{ }_{\lambda} S_{t_{j}}^{h, \pi},{ }_{\lambda} \widehat{Y}_{t_{j}}^{h, n-1, \pi},{ }_{\lambda} \widehat{Z}_{t_{j}}^{h, n-1, \pi}\right) \Delta_{j}\right|^{2}
\end{aligned}
$$

and similarly for $d=1, \ldots, D$.

Next, we derive almost sure convergence of the simulation based estimators starting with the coefficients. 
Lemma 4.4. $\left(\alpha_{0, i}^{h, n, \pi, L}, \ldots, \alpha_{D, i}^{h, n, \pi, L}\right)$ converges P-almost surely to $\left(\alpha_{0, i}^{h, n, \pi}, \ldots, \alpha_{D, i}^{h, n, \pi}\right)$, when L tends to infinity.

Proof. We proceed with an induction on $n$. For $n=0$ the claim is true by definition, since

$$
\alpha_{d, i, k}^{h, 0, \pi, L}=\alpha_{d, i, k}^{h, 0, \pi}=0, \quad d=0, \ldots, D, k=1, \ldots, K_{d, i} .
$$

Now, we suppose convergence is already proved for some $(n-1) \in \mathbb{N}_{0}$. We only show the convergence for $\alpha_{d, i, k}^{h, n, \pi, L}$ for some fixed $d \geq 1$, the arguments for $\alpha_{0, i, k}^{h, n, \pi}$ are basically the same.

By the law of large numbers we have

$$
\lim _{L \rightarrow \infty} \mathcal{B}_{d, i}^{h, L}=\mathcal{B}_{d, i}^{h}, \text { P-a.s. }
$$

As $\mathcal{B}_{d, i}^{h}$ is invertible the same is valid for $\mathcal{B}_{d, i}^{h, L}$ provided $L$ is large enough. We assume $L$ to satisfy this condition in the following. In particular, $\mathcal{A}_{d, i}^{h, L}$ then has full rank and its pseudo-inverse can be written as

$$
\left(\mathcal{A}_{d, i}^{h, L}\right)^{+}=\left(\mathcal{B}_{d, i}^{h, L}\right)^{-1}\left(\mathcal{A}_{d, i}^{h, L}\right)^{\top} .
$$

Hence, we obtain

$$
\begin{aligned}
\alpha_{d, i}^{h, n, \pi, L}=\left(\mathcal{B}_{d, i}^{h, L}\right)^{-1}\left(\frac{1}{L} \sum_{\lambda=1}^{L}{ }_{\lambda} \eta_{d, i}^{h} \frac{\Delta_{\lambda} W_{d, i}^{h, \pi}}{\Delta_{i}}\right. & \left({ }_{\lambda} \Psi_{t_{N}}^{h, \pi, 0} \phi\left({ }_{\lambda} X_{t_{N}}^{h, \pi}\right)\right. \\
& \left.\left.+\sum_{j=i+1}^{N-1}{ }_{\lambda} \Psi_{t_{j}}^{h, \pi, 0} f\left(t_{j},{ }_{\lambda} S_{t_{j}}^{h, \pi},{ }_{\lambda} \widehat{Y}_{t_{j}}^{h, n-1, \pi},{ }_{\lambda} \widehat{Z}_{t_{j}}^{h, n-1, \pi}\right) \Delta_{j}\right)\right)
\end{aligned}
$$

so that due to (10), we only have to show for all $1 \leq l \leq K_{d, i}$

$$
\begin{aligned}
& \frac{1}{L} \sum_{\lambda=1}^{L}{ }_{\lambda} \eta_{d, i, l}^{h} \frac{\Delta_{\lambda} W_{d, i}^{h, \pi}}{\Delta_{i}}\left({ }_{\lambda} \Psi_{t_{N}}^{h, \pi, 0} \phi\left({ }_{\lambda} X_{t_{N}}^{h, \pi}\right)+\sum_{j=i+1}^{N-1}{ }_{\lambda} \Psi_{t_{j}}^{h, \pi, 0} f\left(t_{j},{ }_{\lambda} S_{t_{j}}^{h, \pi},{ }_{\lambda} \widehat{Y}_{t_{j}}^{h, n-1, \pi},{ }_{\lambda} \widehat{Z}_{t_{j}}^{h, n-1, \pi}\right) \Delta_{j}\right) \\
& \longrightarrow E\left[\eta_{d, i, l}^{h} \frac{\Delta W_{d, i}^{h, \pi}}{\Delta_{i}}\left(\Psi_{t_{N}}^{h, \pi, 0} \phi\left(X_{t_{N}}^{h, \pi}\right)+\sum_{j=i+1}^{N-1} \Psi_{t_{j}}^{h, \pi, 0} f\left(t_{j}, S_{t_{j}}^{h, \pi}, \widehat{Y}_{t_{j}}^{h, n-1, \pi}, \widehat{Z}_{t_{i}}^{h, n-1, \pi}\right) \Delta_{j}\right)\right] \text { P-a.s. }
\end{aligned}
$$

To do so, define

$$
{ }_{\lambda} \widetilde{Y}_{t_{i}}^{h, n-1, \pi}=\sum_{k=1}^{K_{0, i}}\left({ }_{\lambda} \Psi_{t_{i}}^{h, \pi, 0}\right)^{-1} \alpha_{0, i, k}^{h, n-1, \pi}{ }_{\lambda} \eta_{0, i, k}^{h}, \quad{ }_{\lambda} \widetilde{Z}_{d, t_{i}}^{h, n-1, \pi}=\sum_{k=1}^{K_{d, i}}\left({ }_{\lambda} \Psi_{t_{i}}^{h, \pi, 0}\right)^{-1} \alpha_{d, i, k}^{h, n-1, \pi}{ }_{\lambda} \eta_{d, i, k}^{h}
$$

and ${ }_{\lambda} \widetilde{Z}_{t_{i}}^{h, n-1, \pi}=\left({ }_{\lambda} \widetilde{Z}_{1, t_{i}}^{h, n-1, \pi}, \ldots,{ }_{\lambda} \widetilde{Z}_{D, t_{i}}^{h, n-1, \pi}\right)^{\top}$.

Note that

$$
{ }_{\lambda} \eta_{d, i, l}^{h} \frac{\Delta_{\lambda} W_{d, i}^{h, \pi}}{\Delta_{i}}\left({ }_{\lambda} \Psi_{t_{N}}^{h, \pi, 0} \phi\left({ }_{\lambda} X_{t_{N}}^{h, \pi}\right)+\sum_{j=i+1}^{N-1}{ }_{\lambda} \Psi_{t_{j}}^{h, \pi, 0} f\left(t_{j},{ }_{\lambda} S_{t_{j}}^{h, \pi},{ }_{\lambda} \widetilde{Y}_{t_{j}}^{h, n-1, \pi},{ }_{\lambda} \widetilde{Z}_{t_{j}}^{h, n-1, \pi}\right) \Delta_{j}\right), \lambda=1, \ldots, L
$$

are independent and identically distributed with the same distribution as

$$
\eta_{d, i, l}^{h} \frac{\Delta W_{d, i}^{h, \pi}}{\Delta_{i}}\left(\Psi_{t_{N}}^{h, \pi, 0} \phi\left(X_{t_{N}}^{h, \pi}\right)+\sum_{j=i+1}^{N-1} \Psi_{t_{j}}^{h, \pi, 0} f\left(t_{j}, S_{t_{j}}^{h, \pi}, \widehat{Y}_{t_{j}}^{h, n-1, \pi}, \widehat{Z}_{t_{j}}^{h, n-1, \pi}\right) \Delta_{j}\right) .
$$


Moreover, it holds that

$$
\begin{aligned}
& E\left[\eta_{d, i, l}^{h} \frac{\Delta W_{d, i}^{h, \pi}}{\Delta_{i}}\left(\Psi_{t_{N}}^{h, \pi, 0} \phi\left(X_{t_{N}}^{h, \pi}\right)+\sum_{j=i+1}^{N-1} \Psi_{t_{j}}^{h, \pi, 0} f\left(t_{j}, S_{t_{j}}^{h, \pi}, \widehat{Y}_{t_{j}}^{h, n-1, \pi}, \widehat{Z}_{t_{j}}^{h, n-1, \pi}\right) \Delta_{j}\right)\right] \\
& \leq \frac{1}{\Delta_{i}} \sqrt{E\left[\left(\eta_{d, i, l}^{h} \Delta W_{d, i}^{h, \pi}\right)^{2}\right]} \\
& \times \sqrt{E\left[\left(\Psi_{t_{N}}^{h, \pi, 0} \phi\left(X_{t_{N}}^{h, \pi}\right)+\sum_{j=i+1}^{N-1} \Psi_{t_{j}}^{h, \pi, 0} f\left(t_{j}, S_{t_{j}}^{h, \pi}, \widehat{Y}_{t_{j}}^{h, n-1, \pi}, \widehat{Z}_{t_{j}}^{h, n-1, \pi}\right) \Delta_{j}\right)^{2}\right]} \\
& \leq \frac{2}{\Delta_{i}} \sqrt{E\left[\left(\eta_{d, i, l}^{h}\right)^{2}\right] \Delta_{i}+C_{h}^{2} E\left[\left(\eta_{d, i, l}^{h}\right)^{2}\right] \Delta_{i}^{2}} \\
& \times\left(E\left[\left(\sum_{j=i+1}^{N-1} \Psi_{t_{j}}^{h, \pi, 0}\left(f\left(t_{j}, S_{t_{j}}^{h, \pi}, \widehat{Y}_{t_{j}}^{h, n-1, \pi}, \widehat{Z}_{t_{j}}^{h, n-1, \pi}\right)-f\left(t_{j}, S_{t_{j}}^{h, \pi}, 0,0\right)\right) \Delta_{j}\right)^{2}\right]\right. \\
& \left.+E\left[\left(\Psi_{t_{N}}^{h, \pi, 0} \phi\left(X_{t_{N}}^{h, \pi}\right)+\sum_{j=i+1}^{N-1} \Psi_{t_{j}}^{h, \pi, 0} f\left(t_{j}, S_{t_{j}}^{h, \pi}, 0,0\right) \Delta_{j}\right)^{2}\right]\right)^{1 / 2} \\
& \leq \frac{2\left(1+C_{h}^{2} \Delta_{i}\right)^{1 / 2}}{\sqrt{\Delta_{i}}} \sqrt{E\left[\left(\eta_{d, i, l}^{h}\right)^{2}\right]} \\
& \times\left(2 T^{2} K^{2} E\left[\max _{i+1 \leq j \leq N-1}\left(\left|\Psi_{t_{j}}^{h, \pi, 0} \widehat{Y}_{t_{j}}^{h, n-1, \pi}\right|^{2}+\left|\Psi_{t_{j}}^{h, \pi, 0} \widehat{Z}_{t_{j}}^{h, n-1, \pi}\right|^{2}\right)\right]\right. \\
& \left.+E\left[\left(\Psi_{t_{N}}^{h, \pi, 0} \phi\left(X_{t_{N}}^{h, \pi}\right)+\sum_{j=i+1}^{N-1} \Psi_{t_{j}}^{h, \pi, 0} f\left(t_{j}, S_{t_{j}}^{h, \pi}, 0,0\right) \Delta_{j}\right)^{2}\right]\right)^{1 / 2} \\
& =\frac{2\left(1+C_{h}^{2} \Delta_{i}\right)^{1 / 2}}{\sqrt{\Delta_{i}}} \sqrt{E\left[\left(\eta_{d, i, l}^{h}\right)^{2}\right]} \\
& \times\left(2 T^{2} K^{2} E\left[\max _{i+1 \leq j \leq N-1}\left(\left|\sum_{k=1}^{K_{0, j}} \alpha_{0, j, k}^{h, n-1, \pi} \eta_{0, j, k}^{h}\right|^{2}+\left.\sum_{d=1}^{D}|| \sum_{k=1}^{K_{d, j}} \alpha_{d, j, k}^{h, n-1, \pi} \eta_{d, j, k}^{h}\right|^{2}\right)\right]\right. \\
& \left.+E\left[\left(\Psi_{t_{N}}^{h, \pi, 0} \phi\left(X_{t_{N}}^{h, \pi}\right)+\sum_{j=i+1}^{N-1} \Psi_{t_{j}}^{h, \pi, 0} f\left(t_{j}, S_{t_{j}}^{h, \pi}, 0,0\right) \Delta_{j}\right)^{2}\right]\right)^{1 / 2}<\infty,
\end{aligned}
$$

where we used Hölder's inequality, the independence of $\Delta W_{i}^{h, \pi}$ and $X_{t_{i}}^{h, \pi}$, the Lipschitz continuity of $f$ and Assumption A 7. Therefore we can apply Kolmogorov's law of large numbers, and deduce that

$$
\begin{aligned}
& \frac{1}{L} \sum_{\lambda=1}^{L}{ }_{\lambda} \eta_{d, i, l}^{h} \frac{\Delta_{\lambda} W_{d, i}^{h, \pi}}{\Delta_{i}}\left({ }_{\lambda} \Psi_{t_{N}}^{h, \pi, 0} \phi\left({ }_{\lambda} X_{t_{N}}^{h, \pi}\right)+\sum_{j=i+1}^{N-1}{ }_{\lambda} \Psi_{t_{j}}^{h, \pi, 0} f\left(t_{j},{ }_{\lambda} S_{t_{j}}^{h, \pi},{ }_{\lambda} \widetilde{Y}_{t_{j}}^{h, n-1, \pi},{ }_{\lambda} \widetilde{Z}_{t_{j}}^{h, n-1, \pi}\right) \Delta_{j}\right) \\
& \longrightarrow E\left[\eta_{d, i, l}^{h} \frac{\Delta W_{d, i}^{h, \pi}}{\Delta_{i}}\left(\Psi_{t_{N}}^{h, \pi, 0} \phi\left(X_{t_{N}}^{h, \pi}\right)+\sum_{j=i+1}^{N-1} \Psi_{t_{j}}^{h, \pi, 0} f\left(t_{j}, S_{t_{j}}^{h, \pi}, \widehat{Y}_{t_{j}}^{h, n-1, \pi}, \widehat{Z}_{t_{j}}^{h, n-1, \pi}\right) \Delta_{j}\right)\right] \text { P-a.s. }
\end{aligned}
$$


Finally, we obtain

$$
\begin{aligned}
& \mid \frac{1}{L} \sum_{\lambda=1}^{L}{ }_{\lambda} \eta_{d, i, l}^{h} \frac{\Delta_{\lambda} W_{d, i}^{h, \pi}}{\Delta_{i}}\left({ }_{\lambda} \Psi_{t_{N}}^{h, \pi, 0} \phi\left({ }_{\lambda} X_{t_{N}}^{h, \pi}\right)+\sum_{j=i+1}^{N-1}{ }_{\lambda} \Psi_{t_{j}}^{h, \pi, 0} f\left(t_{j},{ }_{\lambda} S_{t_{j}}^{h, \pi},{ }_{\lambda} \widehat{Y}_{t_{j}}^{h, n-1, \pi},{ }_{\lambda} \widehat{Z}_{t_{j}}^{h, n-1, \pi}\right) \Delta_{j}\right) \\
& -\frac{1}{L} \sum_{\lambda=1}^{L}{ }_{\lambda} \eta_{d, i, l}^{h} \frac{\Delta_{\lambda} W_{d, i}^{h, \pi}}{\Delta_{i}}\left({ }_{\lambda} \Psi_{t_{N}}^{h, \pi, 0} \phi\left({ }_{\lambda} X_{t_{N}}^{h, \pi}\right)+\sum_{j=i+1}^{N-1}{ }_{\lambda} \Psi_{t_{j}}^{h, \pi, 0} f\left(t_{j}, \lambda_{\lambda} S_{t_{j}}^{h, \pi},{ }_{\lambda} \widetilde{Y}_{t_{j}}^{h, n-1, \pi},{ }_{\lambda} \widetilde{Z}_{t_{j}}^{h, n-1, \pi}\right) \Delta_{j}\right) \mid \\
& \leq \frac{K}{L} \sum_{\lambda=1}^{L}\left|{ }_{\lambda} \eta_{d, i, l}^{h} \frac{\Delta_{\lambda} W_{d, i}^{h, \pi}}{\Delta_{i}}\right| \cdot\left(\sum_{j=i+1}^{N-1}{ }_{\lambda} \Psi_{t_{j}}^{h, \pi}{ }_{\lambda} \widehat{Y}_{t_{j}}^{h, n-1, \pi}-{ }_{\lambda} \widetilde{Y}_{t_{j}}^{h, n-1, \pi} \mid\right. \\
& \left.+{ }_{\lambda} \Psi_{t_{j}}^{h, \pi, 0}\left|{ }_{\lambda} \widehat{Z}_{t_{j}}^{h, n-1, \pi}-{ }_{\lambda} \widetilde{Z}_{t_{j}}^{h, n-1, \pi}\right|\right) \\
& \leq \frac{K}{L} \sum_{\lambda=1}^{L}\left|{ }_{\lambda} \eta_{d, i, l}^{h} \frac{\Delta_{\lambda} W_{d, i}^{h, \pi}}{\Delta_{i}}\right| \cdot \sum_{j=i+1}^{N-1}\left(\left.\sum_{k=1}^{K_{0, j}}\right|_{\lambda} \eta_{0, j, k}^{h}|\cdot| \alpha_{0, j, k}^{h, n-1, \pi, L}-\alpha_{0, j, k}^{h, n-1, \pi} \mid\right. \\
& \left.+\sum_{d=1}^{D} \sum_{k=1}^{K_{d, j}}\left|{ }_{\lambda} \eta_{d, j, k}^{h}\right| \cdot\left|\alpha_{d, j, k}^{h, n-1, \pi, L}-\alpha_{d, j, k}^{h, n-1, \pi}\right|\right) \\
& \leq \max _{0 \leq j \leq N-1}\left(\max _{1 \leq k \leq K_{0, j}}\left|\alpha_{0, j, k}^{h, n-1, \pi, L}-\alpha_{0, j, k}^{h, n-1, \pi}\right|+\max _{1 \leq d \leq D} \max _{1 \leq k \leq K_{d, j}}\left|\alpha_{d, j, k}^{h, n-1, \pi, L}-\alpha_{d, j, k}^{h, n-1, \pi}\right|\right) \\
& \times \frac{K}{L} \sum_{\lambda=1}^{L}\left|{ }_{\lambda} \eta_{d, i, l}^{h} \frac{\Delta_{\lambda} W_{d, i}^{h, \pi}}{\Delta_{i}}\right| \cdot \sum_{j=i+1}^{N-1}\left(\left.\sum_{k=1}^{K_{0, j}}\right|_{\lambda} \eta_{0, j, k}^{h}\left|+\sum_{d=1}^{D} \sum_{k=1}^{K_{d, j}}\right|_{\lambda} \eta_{d, j, k}^{h} \mid\right) .
\end{aligned}
$$

The first factor converges to zero due to the induction hypothesis, the second one to a finite number due to the law of large numbers. Combining (10)-(13) yields the claim.

Consequently, we obtain the $P$-a.s. convergence of the simulation based estimator:

Theorem 4.5. $\left(\widehat{Y}_{t_{i}}^{h, n, \pi, L}, \widehat{Z}_{t_{i}}^{h, n, \pi, L}\right)$ converges P-almost surely to $\left(\widehat{Y}_{t_{i}}^{h, n, \pi}, \widehat{Z}_{t_{i}}^{h, n, \pi}\right)$ as L tends to infinity.

Proof. For any $0 \leq i \leq N$ we obtain

$$
\begin{aligned}
& \left|\widehat{Y}_{t_{i}}^{h, n, \pi, L}-\widehat{Y}_{t_{i}}^{h, n, \pi}\right|+\left|\widehat{Z}_{t_{i}}^{h, n, \pi, L}-\widehat{Z}_{t_{i}}^{h, n, \pi}\right| \\
& =\left|\sum_{k=1}^{K_{0, i}}\left(\alpha_{0, i, k}^{h, n, \pi, L}-\alpha_{0, i, k}^{h, n, \pi}\right)\left(\Psi_{t_{i}}^{h, \pi, 0}\right)^{-1} \eta_{0, i, k}^{h}\right|+\left|\sum_{d=1}^{D} \sum_{k=1}^{K_{d, i}}\left(\alpha_{d, i, k}^{h, n, \pi, L}-\alpha_{d, i, k}^{h, n, \pi}\right)\left(\Psi_{t_{i}}^{h, \pi, 0}\right)^{-1} \eta_{d, i, k}^{h}\right| \\
& \leq \max _{1 \leq k \leq K_{0, i}}\left|\alpha_{0, i, k}^{h, n, \pi, L}-\alpha_{0, i, k}^{h, n, \pi}\right| \sum_{k=1}^{K_{0, i}}\left|p_{0, i, k}\left(X_{t_{i}}^{h, \pi}\right)\right| \\
& \quad+\max _{1 \leq d \leq D} \max _{1 \leq k \leq K_{d, i}}\left|\alpha_{d, i, k}^{h, n, \pi, L}-\alpha_{d, i, k}^{h, n, \pi}\right| \sum_{d=1}^{D} \sum_{k=1}^{K_{d, i}}\left|p_{d, i, k}\left(X_{t_{i}}^{h, \pi}\right)\right| .
\end{aligned}
$$

Hence the claim follows in view of Lemma 4.4.

We now summarize the approximation of $\left(Y_{0}, Z_{0}\right)$ by the modified forward scheme with importance sampling in a least-squares Monte Carlo framework: 
The final estimator for $\left(Y_{0}, Z_{0}\right)$ is $\left(\widehat{Y}_{t_{0}}^{h, n, \pi, L}, \widehat{Z}_{t_{0}}^{h, n, \pi, L}\right)$. Notice that at time $t_{0}=0$ the only choice for the projection spaces is $\Lambda_{d, 0}^{h}=\mathbb{R}$ for any $0 \leq d \leq D$. Taking $\{1\}$ as basis, we observe that

$$
\begin{aligned}
\widehat{Y}_{t_{0}}^{h, n, \pi, L} & =\frac{1}{L} \sum_{\lambda=1}^{L}\left({ }_{\lambda} \Psi_{t_{N}}^{h, \pi, 0} \phi\left({ }_{\lambda} X_{t_{N}}^{h, \pi}\right)+\sum_{j=0}^{N-1}{ }_{\lambda} \Psi_{t_{j}}^{h, \pi, 0} f\left(t_{j},{ }_{\lambda} S_{t_{j}}^{h, \pi},{ }_{\lambda} \widehat{Y}_{t_{j}}^{h, n-1, \pi},{ }_{\lambda} \widehat{Z}_{t_{j}}^{h, n-1, \pi}\right) \Delta_{j}\right) \\
\widehat{Z}_{d, t_{0}}^{h, n, \pi, L} & =\frac{1}{L} \sum_{\lambda=1}^{L} \frac{\Delta_{\lambda} W_{d, 0}^{h, \pi}}{\Delta_{0}}\left({ }_{\lambda} \Psi_{t_{N}}^{h, \pi, 0} \phi\left({ }_{\lambda} X_{t_{N}}^{h, \pi}\right)+\sum_{j=1}^{N-1}{ }_{\lambda} \Psi_{t_{j}}^{h, \pi, 0} f\left(t_{j},{ }_{\lambda} S_{t_{j}}^{h, \pi},{ }_{\lambda} \widehat{Y}_{t_{j}}^{h, n-1, \pi},{ }_{\lambda} \widehat{Z}_{t_{j}}^{h, n-1, \pi}\right) \Delta_{j}\right)
\end{aligned}
$$

It is important to see that the averaging here is over dependent paths, because the weights in the definition of $\left({ }_{\lambda} \widehat{Y}_{t_{j}}^{h, n-1, \pi},{ }_{\lambda} \widehat{Z}_{t_{j}}^{h, n-1, \pi}\right)$ depend on the whole collection of sample path. In the very special case $f=0$, one averages, however, over independent paths and $\widehat{Y}_{t_{0}}^{h, n, \pi, L}$ reduces to the usual Monte Carlo estimator for the expectation of $\phi\left(X_{T}\right)$ with importance sampling given by $h$. In the context of option pricing (with $f=0$ ) in a complete market $Z_{0}$ is (up to a linear transformation) the delta of the option. The estimator $\widehat{Z}_{d, t}^{h, n, \pi}$ then corresponds to the likelihood ratio delta with importance sampling. For more information on this classical situation we refer to Glasserman [13], Ch. 4.6 for importance sampling, and Ch. 7.3 for the likelihood ratio delta.

We now decompose the error into

$$
\begin{aligned}
& \left|Y_{0}-\widehat{Y}_{t_{0}}^{h, n, \pi, L}\right|^{2}+\left|Z_{0}-\widehat{Z}_{t_{0}}^{h, n, \pi, L}\right|^{2} \\
\leq & 3\left(\left|Y_{0}-Y_{t_{0}}^{h, n, \pi}\right|^{2}+\left|Z_{0}-Z_{t_{0}}^{h, n, \pi}\right|^{2}\right)+3\left(\left|Y_{t_{0}}^{h, n, \pi}-\widehat{Y}_{t_{0}}^{h, n, \pi}\right|^{2}+\left|Z_{t_{0}}^{h, n, \pi}-\widehat{Z}_{t_{0}}^{h, n, \pi}\right|^{2}\right) \\
& +3\left(\left|\widehat{Y}_{t_{0}}^{h, n, \pi}-\widehat{Y}_{t_{0}}^{h, n, \pi, L}\right|^{2}+\left|\widehat{Z}_{t_{0}}^{h, n, \pi}-\widehat{Z}_{t_{0}}^{h, n, \pi, L}\right|^{2}\right)
\end{aligned}
$$

The first term captures the error due to the time discretization and the iteration. We know from the results in Section 3 that this error does not depend on the choice of $h$. In typical situations it is of order $1 / 2$ in the mesh size of the time partition and converges exponentially in the number of iterations, see Corollary 3.2. Although the size of this first error term does not depend on $h$, we emphasize that $Y_{t_{0}}^{h, n, \pi}$ is the expectation of an expression, whose variance changes with $h$. The second term contains the error stemming from the choice of the basis. Obviously, the weights $(6)-(7)$ in the construction of $\left(\widehat{Y}_{t_{0}}^{h, n, \pi}, \widehat{Z}_{t_{0}}^{h, n, \pi}\right)$ depend on $h$. Hence, for the second error term, the choice of $h$ influences the error term itself and the variances in the computation of the weights. By Theorem 4.2 the second term converges to zero when the basis increases in a way that the projections spaces $\Lambda_{d, i}^{h}$ exhaust the space $L^{2}\left(\mathcal{G}_{i}^{h, \pi}, P\right)$. Finally, the third term covers the simulation error. Thanks to Theorem 4.5 this error converges to zero almost surely as the number of path tends to infinity. The objective of the importance sampling procedure, introduced in this paper, is to reduce this third error by a judicious choice of $h$. While a theoretical analysis for the choice of $h$ is beyond the scope of the present paper, we illustrate the success of this importance sampling by some numerical examples in the next section.

\section{$5 \quad$ Numerical experiments}

We now apply the algorithm to the pricing problem of an Asian call option on a single stock in a complete market in two different models. The terminal condition in this case is of the form

$$
\phi\left(S_{T}, \int_{0}^{T} S_{u} d u\right)
$$

i.e.

$$
X_{t}=\left(S_{t}, \int_{0}^{t} S_{u} d u\right)
$$


For approximation purposes we replace it by some discrete-time counterpart. Here we choose the simplest approximation

$$
\phi\left(S_{t_{N}}^{\pi}, \sum_{j=0}^{N-1} S_{t_{j}}^{\pi} \Delta_{j}\right)
$$

Corollary 4.4 of Zhang [27] completes the error analysis of Theorem 2.1 and Corollary 3.2: There is a constant $C$ such that

$$
E\left[\left|\phi\left(S_{T}, \int_{0}^{T} S_{u} d u\right)-\phi\left(S_{t_{N}}^{\pi}, \sum_{j=0}^{N-1} S_{t_{j}}^{\pi} \Delta_{j}\right)\right|^{2}\right] \leq C|\pi|
$$

The first model which we consider is the standard Black-Scholes model with constant coefficients. Hence the FBSDE becomes, after the measure transformation,

$$
\begin{aligned}
d S_{t}^{h} & =\left[b S_{t}^{h}+\sigma S_{t}^{h} h_{t}\right] d t+\sigma S_{t}^{h} d W_{t}, \\
d Y_{t}^{h} & =\left[r Y_{t}^{h}+\frac{b-r}{\sigma} Z_{t}^{h}+Z_{t}^{h} h_{t}\right] d t+Z_{t}^{h} d W_{t}, \\
S_{0}^{h} & =s_{0}, \quad Y_{T}^{h}=\left(\frac{1}{T} \int_{0}^{T} S_{t}^{h} d t-K\right)^{+} .
\end{aligned}
$$

Moreover, we consider the model introduced by Bergman [6], where we have different interest rates for lending $r$ and borrowing $R$, resulting in a nonlinear driver of the backward part:

$$
\begin{aligned}
d S_{t}^{h} & =\left[b S_{t}^{h}+\sigma S_{t}^{h} h_{t}\right] d t+\sigma S_{t}^{h} d W_{t}, \\
d Y_{t}^{h} & =\left[r Y_{t}^{h}+\frac{b-r}{\sigma} Z_{t}^{h}-(R-r)\left(Y_{t}^{h}-\frac{Z_{t}^{h}}{\sigma}\right)^{-}+Z_{t}^{h} h_{t}\right] d t+Z_{t}^{h} d W_{t}, \\
S_{0}^{h} & =s_{0}, \quad Y_{T}^{h}=\left(\frac{1}{T} \int_{0}^{T} S_{t}^{h} d t-K\right)^{+} .
\end{aligned}
$$

We now have to specify the discrete process $h_{t_{i}}$ for the importance sampling. In the following we concentrate on the variance induced by the terminal condition and neglect the variance stemming from the driver. Therefore we can make use of the rich literature concerning the choice of $h$, which is available in the context of option pricing. We pick out a rather heuristic approach by Glasserman [13] for this task. Glasserman et al. [12] show that this method is equivalent to another asymptotically optimal approach for the linear problem.

We restrict ourselves to equidistant time increments, i.e. $\Delta_{i}=|\pi|, i=0, \ldots, N-1$, and also $h_{t_{i}}$ will have the simple structure $h_{t_{i}}=\frac{h_{i}}{\sqrt{|\pi|}}$ for $h_{i} \in \mathbb{R}$. Hence, using the Euler-Maruyama scheme, we receive

$$
S_{t_{i+1}}^{h, \pi}=S_{t_{i}}^{h, \pi}+b S_{t_{i}}^{h, \pi}|\pi|+\sigma S_{t_{i}}^{h, \pi} \frac{h_{i}}{\sqrt{|\pi|}}|\pi|+\sigma S_{t_{i}}^{h, \pi} \Delta W_{i}=S_{t_{i}}^{h, \pi}+b S_{t_{i}}^{h, \pi}|\pi|+\sigma S_{t_{i}}^{h, \pi} \sqrt{|\pi|}\left(\xi_{i}+h_{i}\right)
$$

for standard normal random variables $\xi_{i}$. Defining $\xi=\left(\xi_{0}, \ldots, \xi_{N-1}\right)$ and $\mathfrak{h}=\left(h_{0}, \ldots, h_{N-1}\right)$ we also get $\Psi_{t_{N}}^{h, \pi, 0}=\exp \left\{-\mathfrak{h}^{\top} \xi-\frac{1}{2} \mathfrak{h}^{\top} \mathfrak{h}\right\}$

As approximation for the terminal condition we choose $\phi\left(X_{t_{N}}^{h, \pi}\right)=\left(\frac{1}{N+1} \sum_{i=0}^{N} S_{t_{i}}^{h, \pi}-K\right)^{+}$. In abuse of notation we consider $\phi$ as function of $\xi$ so that we can write

$$
\begin{aligned}
E\left[\phi(\xi) \mathbf{1}_{\phi(\xi)>0}\right] & =E\left[\exp \{\ln (\phi(\xi))\} \mathbf{1}_{\phi(\xi)>0}\right] \\
& =E\left[\exp \{\ln (\phi(\xi+\mathfrak{h}))\} \exp \left\{-\mathfrak{h}^{\top} \xi-\frac{1}{2} \mathfrak{h}^{\top} \mathfrak{h}\right\} \mathbf{1}_{\phi(\xi)>0}\right] \\
& \approx E\left[\exp \left\{\ln (\phi(\mathfrak{h}))+\nabla \ln (\phi(\mathfrak{h})) \xi-\mathfrak{h}^{\top} \xi-\frac{1}{2} \mathfrak{h}^{\top} \mathfrak{h}\right\} \mathbf{1}_{\phi(\xi)>0}\right]
\end{aligned}
$$




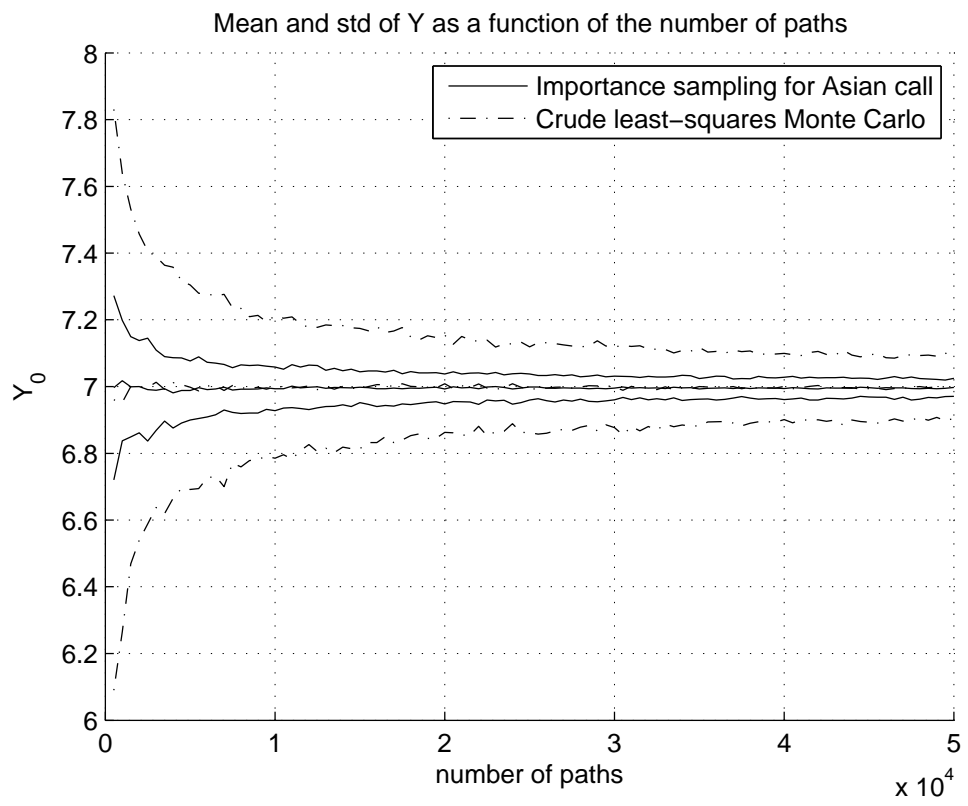

Figure 1: Convergence of $\widehat{Y}_{t_{0}}^{h, n_{\text {stop }}, \pi, L}$ in the case of linear BSDE.

If one can find $\mathfrak{h}$ which satisfies $\nabla \ln (\phi(\mathfrak{h}))=\mathfrak{h}^{\top}$, we would approximatively end up with the expectation of a constant random variable independent of $\xi$ and consequently get a low-variance estimator. The condition $\nabla \ln (\phi(\mathfrak{h}))=\mathfrak{h}^{\top}$ is the necessary condition for a local maximum of the function $\mathfrak{h} \mapsto \ln (\phi(\mathfrak{h}))-\frac{1}{2} \mathfrak{h}^{\top} \mathfrak{h}$. However, maximizing this function is equivalent to maximizing $\phi(\mathfrak{h}) \exp \left(-\frac{1}{2} \mathfrak{h}^{\top} \mathfrak{h}\right)$. This can be done numerically as described by Glasserman [13].

In our simulations we use 20 time steps and a function basis of bivariate monomials $x_{1}^{\alpha}, x_{2}^{\beta}$ for $0 \leq$ $\alpha, \beta \leq 3$ for every point in the time grid. That is we choose $K_{0, i}=K_{1, i}=16$ and $p_{0, i}(\cdot)=p_{1, i}(\cdot)$ for $i=1, \ldots, N-1$. Choosing the same basis for the $Y$ - and the $Z$-component, we can use the same matrices for several computations thereby reducing considerably the computation time. The Picard-iteration is executed until the difference of two subsequent estimators of the initial price is smaller than the stop condition 0.001 i.e. $\left|\widehat{Y}_{t_{0}}^{h, n, \pi, L}-\widehat{Y}_{t_{0}}^{h, n-1, \pi, L}\right|<0.001$. In the option pricing problems of this section the number of executed Picard iterations is rather low. There are no more than 4-6 iterations necessary to get this accuracy.

The following parameters are used for the simulation procedure:

\begin{tabular}{cccccc}
\hline $\mathrm{b}$ & $\sigma$ & $\mathrm{r}$ & $\mathrm{T}$ & $s_{0}$ & $\mathrm{~K}$ \\
\hline 0.06 & 0.2 & 0.1 & 1 & 100 & 100 \\
\hline
\end{tabular}

This example was also examined by Gobet et al. [14] without variance reduction in the context of the backward scheme. As reference value Lapeyre and Temam [21] give 7.04 for the linear BSDE.

We calculate the estimators for the initial price for a number of simulations from 500 up to 50.000 and repeat this procedure 100 times, thereby examining the convergence behavior with respect to the number of simulations. Figure 1 shows the empirical mean plus/minus two empirical standard deviations of the 100 independent repetitions for the estimated initial value $\widehat{Y}_{t_{0}}^{h, n_{\mathrm{stop}}, \pi, L}$ in the Black-Scholes model using the crude least-squares Monte Carlo method and the variance reduced algorithm respectively. We see that importance sampling reduces the empirical variance roughly by a factor of 11, which demonstrates the success of the variance reduction. The estimation of the price is at approximately 7.00 slightly below the reference value of 7.04. This error is mainly due to the crude time discretization of the average. It 


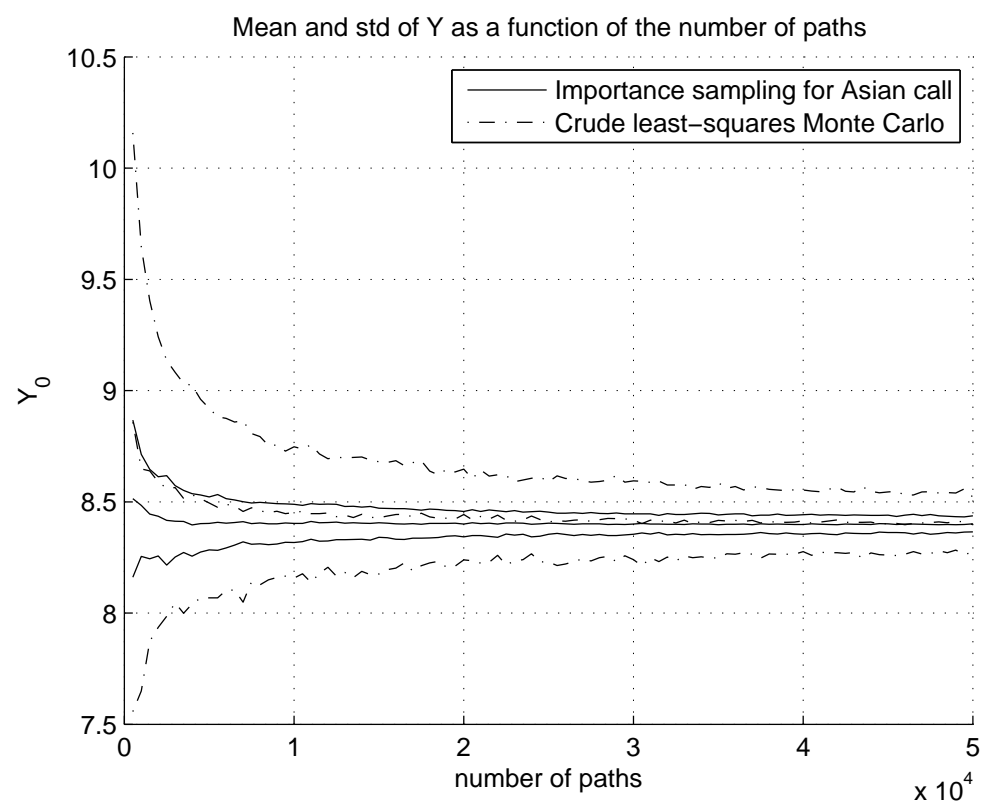

Figure 2: Convergence of $\widehat{Y}_{t_{0}}^{h, n_{\text {stop }}, \pi, L}$ in the case of nonlinear BSDE.

can be mended by adding more time steps or applying more sophisticated discretization schemes for the average as suggested by Lapeyre and Temam [21] or Jourdain and Sbai [17].

We get a similar result concerning the empirical variance, see Figure 2, in the case of different interest rates for lending and borrowing. Here, we use $R=0.15, r=0.1$ and all the other parameters stay the same. This method is even more effective in the out-of-the-money case. We illustrate this with Figure 3 , where we again take the model with the nonlinear BSDE but now with a higher strike $(K=120)$. In this case we receive an empirical variance reduction by a factor of 36 in average.

Overall we may conclude that in the test examples on Asian option pricing the introduction of importance sampling drastically increases the efficiency of the Picard-type scheme for BSDEs.

\section{A Proof of Lemma 3.5}

Define $y_{t_{i}}:=y_{t_{i}}^{(1)}-y_{t_{i}}^{(2)}, z_{t_{i}}:=z_{t_{i}}^{(1)}-z_{t_{i}}^{(2)}$ and $\Delta f_{i}:=f\left(t_{i}, S_{t_{i}}^{h, \pi}, y_{t_{i}}^{(1)}, z_{t_{i}}^{(1)}\right)-f\left(t_{i}, S_{t_{i}}^{h, \pi}, y_{t_{i}}^{(2)}, z_{t_{i}}^{(2)}\right)$. Since $\Psi_{t_{j}}^{h, \pi, 0}$ is an $\left\{\mathcal{F}_{t_{j}}\right\}$-martingale, we can rewrite, noting (1) and (2),

$$
\begin{aligned}
\Psi_{t_{i}}^{h, \pi, 0} Y_{t_{i}}^{(\iota)}= & E\left[\Psi_{t_{N}}^{h, \pi, 0}\left(\phi\left(X_{t_{N}}^{h, \pi}\right)+\sum_{j=i+1}^{N-1} f\left(t_{j}, S_{t_{j}}^{h, \pi}, y_{t_{j}}^{(\iota)}, z_{t_{j}}^{(\iota)}\right) \Delta_{j}\right) \mid \mathcal{F}_{t_{i}}\right] \\
& +E\left[\Psi_{t_{N}}^{h, \pi, 0} \mid \mathcal{F}_{t_{i}}\right] f\left(t_{i}, S_{t_{i}}^{h, \pi}, y_{t_{i}}^{(\iota)}, z_{t_{i}}^{(\iota)} \Delta_{i}\right. \\
= & E\left[\Psi_{t_{i+1}}^{h, \pi, 0} Y_{t_{i+1}}^{(\iota)} \mid \mathcal{F}_{t_{i}}\right]+\Psi_{t_{i}}^{h, \pi, 0} f\left(t_{i}, S_{t_{i}}^{h, \pi}, y_{t_{i}}^{(\iota)}, z_{t_{i}}^{(\iota)}\right) \Delta_{i}
\end{aligned}
$$

and

$$
\Psi_{t_{i}}^{h, \pi, 0} Z_{d, t_{i}}^{(\iota)}=E\left[\Psi_{t_{i+1}}^{h, \pi, 0} \frac{\Delta W_{d, i}^{h, \pi}}{\Delta_{i}} Y_{t_{i+1}}^{(\iota)} \mid \mathcal{F}_{t_{i}}\right]
$$




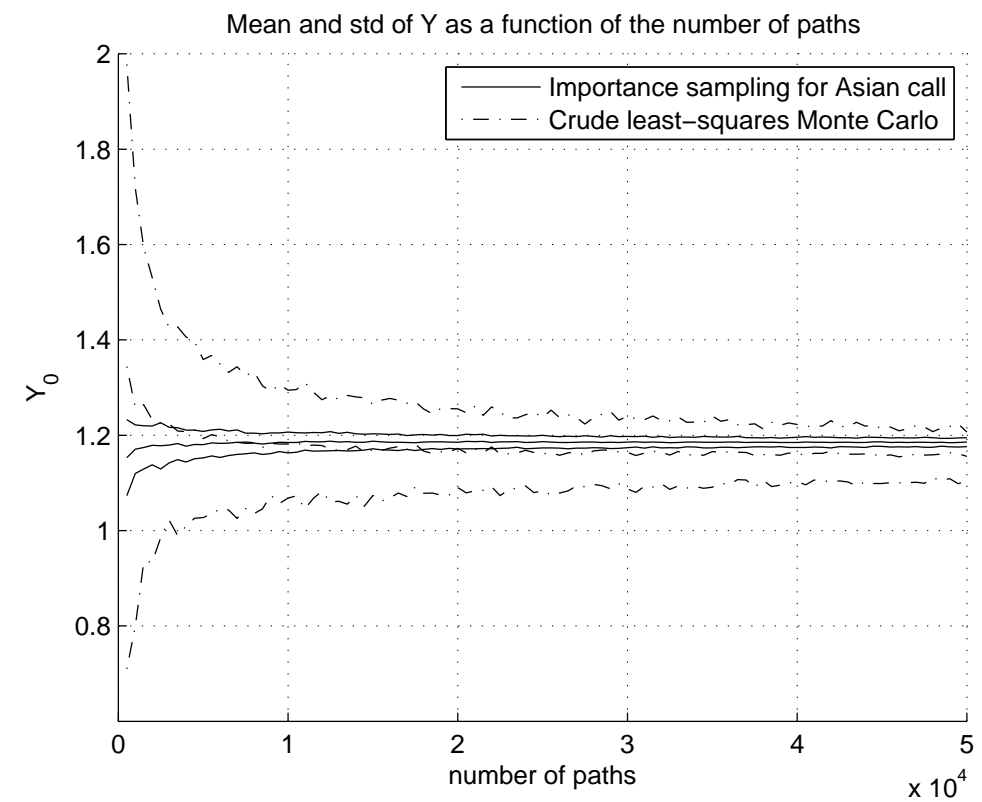

Figure 3: Convergence of $\widehat{Y}_{t_{0}}^{h, n_{\mathrm{stop}}, \pi, L}$ in the case of nonlinear BSDE and out of the money option.

In a first step we show that

$$
\begin{aligned}
& \sum_{i=0}^{N-1} \lambda_{i} E\left[\left|\Psi_{t_{i}}^{h, \pi, 0} Z_{d, t_{i}}^{(1)}-\Psi_{t_{i}}^{h, \pi, 0} Z_{d, t_{i}}^{(2)}\right|^{2}\right] \Delta_{i} \\
\leq & 2\left(\gamma+C_{h}^{2}\right) \sum_{i=0}^{N-1} \lambda_{i} E\left[\left|\Psi_{t_{i}}^{h, \pi, 0} Y_{t_{i}}^{(1)}-\Psi_{t_{i}}^{h, \pi, 0} Y_{t_{i}}^{(2)}\right|^{2}\right] \Delta_{i}+\frac{2(T+1) K^{2}}{\gamma} \sum_{i=0}^{N-1} \lambda_{i} E\left[\left|\Psi_{t_{i}}^{h, \pi, 0} z_{t_{i}}\right|^{2}\right] \Delta_{i} \\
& +\frac{2(T+1) K^{2}}{T \gamma} \sum_{i=0}^{N-1} \lambda_{i} E\left[\left|\Psi_{t_{i}}^{h, \pi, 0} y_{t_{i}}\right|^{2}\right] \Delta_{i} .
\end{aligned}
$$

We have

$$
\begin{aligned}
& \Psi_{t_{i}}^{h, \pi, 0} Z_{d, t_{i}}^{(1)}-\Psi_{t_{i}}^{h, \pi, 0} Z_{d, t_{i}}^{(2)}=E\left[\frac{\Delta W_{d, i}+h_{t_{i}} \Delta_{i}}{\Delta_{i}}\left(\Psi_{t_{i+1}}^{h, \pi, 0} Y_{t_{i+1}}^{(1)}-\Psi_{t_{i+1}}^{h, \pi, 0} Y_{t_{i+1}}^{(2)}\right) \mid \mathcal{F}_{t_{i}}\right] \\
& =E\left[\frac{\Delta W_{d, i}}{\Delta_{i}}\left(\Psi_{t_{i+1}}^{h, \pi, 0} Y_{t_{i+1}}^{(1)}-\Psi_{t_{i+1}}^{h, \pi, 0} Y_{t_{i+1}}^{(2)}-E\left[\Psi_{t_{i+1}}^{h, \pi, 0} Y_{t_{i+1}}^{(1)}-\Psi_{t_{i+1}}^{h, \pi, 0} Y_{t_{i+1}}^{(2)} \mid \mathcal{F}_{t_{i}}\right]\right) \mid \mathcal{F}_{t_{i}}\right] \\
& \quad+E\left[h_{t_{i}}\left(\Psi_{t_{i+1}}^{h, \pi, 0} Y_{t_{i+1}}^{(1)}-\Psi_{t_{i+1}}^{h, \pi, 0} Y_{t_{i+1}}^{(2)}\right) \mid \mathcal{F}_{t_{i}}\right] \\
& \leq \sqrt{\frac{1}{\Delta_{i}}} E\left[\left(\Psi_{t_{i+1}}^{h, \pi, 0} Y_{t_{i+1}}^{(1)}-\Psi_{t_{i+1}}^{h, \pi, 0} Y_{t_{i+1}}^{(2)}-E\left[\Psi_{t_{i+1}}^{h, \pi, 0} Y_{t_{i+1}}^{(1)}-\Psi_{t_{i+1}}^{h, \pi, 0} Y_{t_{i+1}}^{(2)} \mid \mathcal{F}_{t_{i}}\right]\right)^{2} \mid \mathcal{F}_{t_{i}}\right]^{1 / 2} \\
& \quad+E\left[h_{t_{i}}\left(\Psi_{t_{i+1}}^{h, \pi, 0} Y_{t_{i+1}}^{(1)}-\Psi_{t_{i+1}}^{h, \pi, 0} Y_{t_{i+1}}^{(2)}\right) \mid \mathcal{F}_{t_{i}}\right] .
\end{aligned}
$$


Hence,

$$
\begin{aligned}
E & {\left[\left|\Psi_{t_{i}}^{h, \pi, 0} Z_{d, t_{i}}^{(1)}-\Psi_{t_{i}}^{h, \pi} Z_{d, t_{i}}^{(2)}\right|^{2}\right] } \\
\leq & \frac{2}{\Delta_{i}} E\left[\left|\Psi_{t_{i+1}}^{h, \pi, 0} Y_{t_{i+1}}^{(1)}-\Psi_{t_{i+1}}^{h, \pi, 0} Y_{t_{i+1}}^{(2)}\right|^{2}-E\left[\Psi_{t_{i+1}}^{h, \pi, 0} Y_{t_{i+1}}^{(1)}-\Psi_{t_{i+1}}^{h, \pi, 0} Y_{t_{i+1}}^{(2)} \mid \mathcal{F}_{t_{i}}\right]^{2}\right] \\
& +2 C_{h}^{2} E\left[\left|\Psi_{t_{i+1}}^{h, \pi, 0} Y_{t_{i+1}}^{(1)}-\Psi_{t_{i+1}}^{h, \pi, 0} Y_{t_{i+1}}^{(2)}\right|^{2}\right] \\
= & \frac{2}{\Delta_{i}} E\left[\left|\Psi_{t_{i+1}}^{h, \pi, 0} Y_{t_{i+1}}^{(1)}-\Psi_{t_{i+1}}^{h, \pi, 0} Y_{t_{i+1}}^{(2)}\right|^{2}-\left|\Psi_{t_{i}}^{h, \pi, 0} Y_{t_{i}}^{(1)}-\Psi_{t_{i}}^{h, \pi, 0} Y_{t_{i}}^{(2)}-\Psi_{t_{i}}^{h, \pi, 0} \Delta f_{i} \Delta_{i}\right|^{2}\right] \\
& +2 C_{h}^{2} E\left[\left|\Psi_{t_{i+1}}^{h, \pi, 0} Y_{t_{i+1}}^{(1)}-\Psi_{t_{i+1}}^{h, \pi, 0} Y_{t_{i+1}}^{(2)}\right|^{2}\right] \\
\leq & \frac{2}{\Delta_{i}} E\left[\left|\Psi_{t_{i+1}}^{h, \pi, 0} Y_{t_{i+1}}^{(1)}-\Psi_{t_{i+1}}^{h, \pi, 0} Y_{t_{i+1}}^{(2)}\right|^{2}-\left|\Psi_{t_{i}}^{h, \pi, 0} Y_{t_{i}}^{(1)}-\Psi_{t_{i}}^{h, \pi, 0} Y_{t_{i}}^{(2)}\right|^{2}\right] \\
& +4 E\left[\left(\Psi_{t_{i}}^{h, \pi, 0} Y_{t_{i}}^{(1)}-\Psi_{t_{i}}^{h, \pi, 0} Y_{t_{i}}^{(2)}\right) \Psi_{t_{i}}^{h, \pi, 0} \Delta f_{i}\right]+2 C_{h}^{2} E\left[\left|\Psi_{t_{i+1}}^{h, \pi, 0} Y_{t_{i+1}}^{(1)}-\Psi_{t_{i+1}}^{h, \pi, 0} Y_{t_{i+1}}^{(2)}\right|^{2}\right] .
\end{aligned}
$$

Multiplying with $\lambda_{i} \Delta_{i}$ and summing up from 0 to $N-1$ yields for $\gamma>0$ :

$$
\begin{aligned}
& \sum_{i=0}^{N-1} \lambda_{i} E\left[\left|\Psi_{t_{i}}^{h, \pi, 0} Z_{d, t_{i}}^{(1)}-\Psi_{t_{i}}^{h, \pi, 0} Z_{d, t_{i}}^{(2)}\right|^{2}\right] \Delta_{i}+2 \lambda_{0} E\left[\left|Y_{t_{0}}^{(1)}-Y_{t_{0}}^{(2)}\right|^{2}\right] \\
& \leq 2 \lambda_{N} E\left[\left|\Psi_{t_{N}}^{h, \pi, 0} Y_{t_{N}}^{(1)}-\Psi_{t_{N}}^{h, \pi, 0} Y_{t_{N}}^{(2)}\right|^{2}\right]+4 \sum_{i=0}^{N-1} \lambda_{i} E\left[\left(\Psi_{t_{i}}^{h, \pi, 0} Y_{t_{i}}^{(1)}-\Psi_{t_{i}}^{h, \pi, 0} Y_{t_{i}}^{(2)}\right) \Psi_{t_{i}}^{h, \pi, 0} \Delta f_{i}\right] \Delta_{i} \\
&+2 C_{h}^{2} \sum_{i=0}^{N-1} \lambda_{i} E\left[\left|\Psi_{t_{i+1}}^{h, \pi, 0} Y_{t_{i+1}}^{(1)}-\Psi_{t_{i+1}}^{h, \pi, 0} Y_{t_{i+1}}^{(2)}\right|^{2}\right] \Delta_{i} \\
& \leq 2 \gamma \sum_{i=0}^{N-1} \lambda_{i} E\left[\left|\Psi_{t_{i}}^{h, \pi, 0} Y_{t_{i}}^{(1)}-\Psi_{t_{i}}^{h, \pi, 0} Y_{t_{i}}^{(2)}\right|^{2}\right] \Delta_{i}+2 \frac{K^{2}}{\gamma} \sum_{i=0}^{N-1} \lambda_{i} E\left[\left(\left|\Psi_{t_{i}}^{h, \pi, 0} y_{t_{i}}\right|+\left|\Psi_{t_{i}}^{h, \pi, 0} z_{t_{i}}\right|\right)^{2}\right] \Delta_{i} \\
&+2 C_{h}^{2} \sum_{i=0}^{N-1} \lambda_{i} E\left[\left|\Psi_{t_{i}}^{h, \pi, 0} Y_{t_{i}}^{(1)}-\Psi_{t_{i}}^{h, \pi, 0} Y_{t_{i}}^{(2)}\right|^{2}\right] \Delta_{i} \\
& \leq 2\left(\gamma+C_{h}^{2}\right) \sum_{i=0}^{N-1} \lambda_{i} E\left[\left|\Psi_{t_{i}}^{h, \pi, 0} Y_{t_{i}}^{(1)}-\Psi_{t_{i}}^{h, \pi, 0} Y_{t_{i}}^{(2)}\right|^{2}\right] \Delta_{i} \\
&+2 \frac{(1+T) K^{2}}{\gamma} \sum_{i=0}^{N-1} \lambda_{i} E\left[\left|\Psi_{t_{i}}^{h, \pi, 0} z_{t_{i}}\right|^{2}\right] \Delta_{i}+2 \frac{(1+T) K^{2}}{T \gamma} \sum_{i=0}^{N-1} \lambda_{i} E\left[\left|\Psi_{t_{i}}^{h, \pi, 0} y_{t_{i}}\right|^{2}\right] \Delta_{i},
\end{aligned}
$$

where we used Young's inequality and $Y_{t_{N}}^{(1)}=Y_{t_{N}}^{(2)}$.

Secondly, we shall derive

$$
\begin{aligned}
& \max _{0 \leq i \leq N} \lambda_{i} E\left[\left|\Psi_{t_{i}}^{h, \pi, 0} Y_{t_{i}}^{(1)}-\Psi_{t_{i}}^{h, \pi, 0} Y_{t_{i}}^{(2)}\right|^{2}\right] \\
& \leq K^{2}(T+1)\left(|\pi|+\frac{1}{\Gamma}\right)\left(\sum_{i=0}^{N-1} \lambda_{i} E\left[\left|\Psi_{t_{i}}^{h, \pi, 0} z_{t_{i}}\right|^{2}\right] \Delta_{i}+\frac{1}{T} \sum_{i=0}^{N-1} \lambda_{i} E\left[\left|\Psi_{t_{i}}^{h, \pi, 0} y_{t_{i}}\right|^{2}\right] \Delta_{i}\right) .
\end{aligned}
$$


To this end we estimate

$$
\begin{aligned}
& E\left[\left|\Psi_{t_{i}}^{h, \pi, 0} Y_{t_{i}}^{(1)}-\Psi_{t_{i}}^{h, \pi, 0} Y_{t_{i}}^{(2)}\right|^{2}\right] \\
& \leq\left(1+\Gamma \Delta_{i}\right) E\left[\left|\Psi_{t_{i+1}}^{h, \pi, 0} Y_{t_{i+1}}^{(1)}-\Psi_{t_{i+1}}^{h, \pi, 0} Y_{t_{i+1}}^{(2)}\right|^{2}\right]+\left(\Delta_{i}+\frac{1}{\Gamma}\right) E\left[\left|\Psi_{t_{i}}^{h, \pi, 0} \Delta f_{i}\right|^{2}\right] \Delta_{i} \\
& \leq\left(1+\Gamma \Delta_{i}\right) E\left[\left|\Psi_{t_{i+1}}^{h, \pi, 0} Y_{t_{i+1}}^{(1)}-\Psi_{t_{i+1}}^{h, \pi, 0} Y_{t_{i+1}}^{(2)}\right|^{2}\right]+\left(|\pi|+\frac{1}{\Gamma}\right) K^{2}(T+1) E\left[\left|\Psi_{t_{i}}^{h, \pi, 0} z_{t_{i}}\right|^{2}\right] \Delta_{i} \\
& \quad+\left(|\pi|+\frac{1}{\Gamma}\right) K^{2} \frac{(T+1)}{T} E\left[\left|\Psi_{t_{i}}^{h, \pi, 0} y_{t_{i}}\right|^{2}\right] \Delta_{i} .
\end{aligned}
$$

Multiplying with $\lambda_{i}$, an iterated application of the above inequality yields (15).

Finally, we put (14)-(15) together, and, noting that $Z$ is $D$-dimensional, we deduce

$$
\begin{aligned}
\max _{0 \leq i \leq N} \lambda_{i} E\left[\left|\Psi_{t_{i}}^{h, \pi, 0} Y_{t_{i}}^{(1)}-\Psi_{t_{i}}^{h, \pi, 0} Y_{t_{i}}^{(2)}\right|^{2}\right]+\sum_{i=0}^{N-1} \lambda_{i} E\left[\left|\Psi_{t_{i}}^{h, \pi, 0} Z_{t_{i}}^{(1)}-\Psi_{t_{i}}^{h, \pi, 0} Z_{t_{i}}^{(2)}\right|^{2}\right] \Delta_{i} \\
\leq K^{2}(T+1)\left(|\pi|+\frac{1}{\Gamma}\right)\left(\sum_{i=0}^{N-1} \lambda_{i} E\left[\left|\Psi_{t_{i}}^{h, \pi, 0} z_{t_{i}}\right|^{2}\right] \Delta_{i}+\frac{1}{T} \sum_{i=0}^{N-1} \lambda_{i} E\left[\left|\Psi_{t_{i}}^{h, \pi, 0} y_{t_{i}}\right|^{2}\right] \Delta_{i}\right) \\
\quad+2 D\left(\gamma+C_{h}^{2}\right) \sum_{i=0}^{N-1} \lambda_{i} E\left[\left|\Psi_{t_{i}}^{h, \pi, 0} Y_{t_{i}}^{(1)}-\Psi_{t_{i}}^{h, \pi, 0} Y_{t_{i}}^{(2)}\right|^{2}\right] \Delta_{i}+\frac{2 D(T+1) K^{2}}{\gamma} \sum_{i=0}^{N-1} \lambda_{i} E\left[\left|\Psi_{t_{i}}^{h, \pi, 0} z_{t_{i}}\right|^{2}\right] \Delta_{i} \\
\quad+\frac{2 D(T+1) K^{2}}{T \gamma} \sum_{i=0}^{N-1} \lambda_{i} E\left[\left|\Psi_{t_{i}}^{h, \pi, 0} y_{t_{i}}\right|^{2}\right] \Delta_{i} \\
\leq K^{2}(T+1)\left(\left(|\pi|+\frac{1}{\Gamma}\right)\left(2 D\left(\gamma+C_{h}^{2}\right) T+1\right)+2 \frac{D}{\gamma}\right) \\
\quad \times\left(\sum_{i=0}^{N-1} \lambda_{i} E\left[\left|\Psi_{t_{i}}^{h, \pi, 0} z_{t_{i}}\right|^{2}\right] \Delta_{i}+\frac{1}{T} \sum_{i=0}^{N-1} \lambda_{i} E\left[\left|\Psi_{t_{i}}^{h, \pi, 0} y_{t_{i}}\right|^{2}\right] \Delta_{i}\right)
\end{aligned}
$$

\section{Acknowledgements}

Thilo Moseler was supported by the DFG (Deutsche Forschungsgemeinschaft) as member of the research unit 518 'Price, Liquidity and Credit Risks: Measurement and Distribution'. He is also grateful to Robert Denk for attracting his attention to this problem and for many fruitful discussions.

Christian Bender gratefully acknowledges partial support by the DFG priority programme 1324 'Mathematische Methoden zur Extraktion quantifizierbarer Information aus komplexen Systemen'.

\section{References}

[1] Bally, V., Pagès, G.: A quantization algorithm for solving discrete time multidimensional optimal stopping problems. Bernoulli 9 (2003), 1003-1049.

[2] Becherer, D.: Bounded solutions to backward SDE's with jumps for utility optimization and indifference hedging. The Annals of Applied Probability 16 No. 4 (2006), 2027-2054.

[3] Belomestny, D., Bender, C., Schoenmakers, J. G. M.: True upper bounds for Bermudan products via non- nested Monte Carlo. Mathematical Finance (2008), forthcoming.

[4] Bender, C., Denk, R.: A forward scheme for backward SDEs. Stochastic Processes and their Applications 117 No. 12 (2007), 1793-1812. 
[5] Bender, C., Zhang, J.: Time discretization and Markovian iteration for coupled FBSDEs. The Annals of Applied Probability 18 No. 1 (2008), 143-177.

[6] Bergman, Y. Z.: Option pricing with differential interest rates. The Review of Financial Studies 8 (1995), 475-500.

[7] Bouchard, B., Touzi, N.: Discrete-time approximation and Monte Carlo simulation of backward stochastic differential equations. Stochastic Processes and their Applications 111 (2004), 175-206.

[8] Boyle, P., Broadie, M., Glasserman, P.: Monte Carlo methods for security pricing. Journal of Economic Dynamics and Control 21 No. 8-9 (1997), 1267-1321.

[9] Carrière, J. F.: Valuation of the early-exercise price for options using simulations and nonparametric regression. Insurance: Mathematics and Economics 19 (1996), 19-30.

[10] Delarue, F., Menozzi, S.: A forward backward stochastic algorithm for quasi-linear PDEs. The Annals of Applied Probability 16 (2006), 140-184.

[11] El Karoui, N., Peng, S., Quenez, M. C.: Backward stochastic differential equations in finance. Mathematical Finance 7 (1997), 1-71.

[12] Glasserman, P., Heidelberger, P., Shahabuddin, P.: Asymptotically optimal importance sampling and stratification for pricing path-dependent options. Mathematical Finance 9 No. 2 (1999), 117152.

[13] Glasserman, P.: Monte Carlo Methods in Financial Engineering, Springer, New York (2004).

[14] Gobet, E., Lemor, J., Warin, X.: A regression-based Monte-Carlo method to solve backward stochastic differential equations. Annals of Applied Probability 15 No. 3 (2005), 2172-2002.

[15] Griffiths, C., Jenkins, P. A., Song, Y. S.: Importance sampling and the two-locus model with subdivided population structure. Advances in Applied Probability 40 No. 2 (2008), 473-500.

[16] Guasoni, P., Robertson, S.: Optimal importance sampling with explicit formulas in continuous time. Finance and Stochastics 12 No. 1 (2008), 1-19.

[17] Jourdain, B., Sbai, M.: Exact retrospective Monte Carlo computation of arithmetic average Asian options. Monte Carlo Methods and Applications 13 No. 2 (2007), 135-171.

[18] Labart, C.: EDSR: analyse de discrétisation et résolution par méthodes de Monte Carlo adaptatives; Perturbation de domaines pour les options américaines. Phd thesis, L'Ecole Polytechnique (2007).

[19] Lemor, J., Gobet, E., Warin, X.: Rate of convergence of an empirical regression method for solving generalized backward stochastic differential equations. Bernoulli 12 No. 5 (2006), 889-916.

[20] Longstaff, F. A., Schwartz, E. S.: Valuing American options by simulation: A simple least-squares approach. Review of Financial Studies 14 (2001), 113-147.

[21] Lapeyre, B., Temam, E.: Competitive Monte Carlo methods for the pricing of Asian options. Journal of Computational Finance 5 (2001), 39-59.

[22] Milstein, G. N., Schoenmakers, J. G. M.: Monte Carlo construction of hedging strategies against multi-asset European claims Stochastics and Stochastics Reports 73 No. 1-2 (2002), 125-157.

[23] Newton, N. J.: Variance reduction for simulated diffusions. SIAM Journal on Applied Mathematics 54 No. 6 (1994), 1780-1805. 
[24] Ökten, G., Salta, E., Göncü, A.: On pricing discrete barrier options using conditional expectation and importance sampling Monte Carlo. Mathematical and Computer Modelling 47 (2008), 484-494.

[25] Schoenmakers, J. G. M., Heemink, A. W., Kloeden, P. E., Ponnambalam, K.: Variance reduction for Monte Carlo simulation of stochastic environmental models. Applied Mathematical Modelling 26 No. 8 (2002), 785-795.

[26] Shirley, P., Edwards, D., Boulos, S.: Monte Carlo and quasi-Monte Carlo methods for computer graphics. In Heinrich, S., Keller, A., Niederreiter, H. (Eds.), Monte Carlo and Quasi-Monte Carlo 2006, Selected papers based on the presentations at the 7th international conference 'Monte Carlo and quasi-Monte Carlo methods in scientific computing', Ulm, Germany, August 14-18, 2006, Springer, Berlin (2007), 167-177.

[27] Zhang, J.: A numerical scheme for BSDEs. The Annals of Applied Probability 14 No. 1 (2004), 459-488. 